\title{
CRISPR/Cas9 targeting Ttc30a mimics ciliary chondrodysplasia with polycystic kidney disease.
}

\author{
Maike Getwan ${ }^{1,2}$, Anselm Hoppmann ${ }^{3}$, Pascal Schlosser ${ }^{3}$, Kelli Grand ${ }^{1,2}$, Weiting Song ${ }^{1}$, Rebecca \\ Diehl$^{1}$, Sophie Schroda ${ }^{1}$, Florian Heeg ${ }^{1}$, Konstantin Deutsch ${ }^{4}$, Friedhelm Hildebrandt ${ }^{4}$, Ekkehart \\ Lausch $^{5}$, Anna Köttgen ${ }^{3}$, Soeren S. Lienkamp ${ }^{1,2 *}$ \\ ${ }^{1}$ Renal Division, Department of Medicine, Faculty of Medicine and Medical Center-University of Freiburg, Freiburg, \\ Germany. \\ 2 Institute of Anatomy and Zurich Center for Integrative Human Physiology (ZIHP), University of Zurich, Zurich, \\ Switzerland \\ ${ }^{3}$ Institute of Genetic Epidemiology, Faculty of Medicine and Medical Center - University of Freiburg, Freiburg, \\ Germany. \\ ${ }^{4}$ Department of Pediatrics, Boston Children's Hospital, Harvard Medical School, Boston, USA \\ 5 Department of Pediatrics, Medical Center-University of Freiburg, Faculty of Medicine, University of Freiburg, \\ Freiburg, \\ Germany.
}

*Correspondence should be addressed to: soeren.lienkamp@uzh.ch

\begin{abstract}
:
Skeletal ciliopathies (e.g. Jeune syndrome, short rib polydactyly syndrome, Sensenbrenner syndrome) are frequently associated with cystic kidney disease and other organ manifestations, but a common molecular mechanism has remained elusive.

We established two models for skeletal ciliopathies (ift80 and ift172) in Xenopus tropicalis, which exhibited severe limb deformities, polydactyly, cystic kidneys, and ciliogenesis defects, closely matching the phenotype of affected patients.

Employing data-mining and an in silico screen we identified candidate genes with similar molecular properties to genetically validated skeletal ciliopathy genes. Among four genes experimentally validated, CRISPR/Cas9 targeting of ttc30a replicated all aspects of the phenotypes observed in the models of genetically confirmed disease genes, including ciliary defects, limb deformations and cystic kidney disease.

Our findings establish three new models for skeletal ciliopathies (ift80, ift172, ttc30a) and identify TTC30A/B as an essential node in the network of ciliary chondrodysplasia and nephronophthisis-like disease proteins implicating post-translational tubulin modifications in its pathogenesis.
\end{abstract}




\section{Introduction}

Primary cilia are hair-like appendages that extend from the surface of most differentiated cells. Their functional impairment or loss results in ciliopathies ${ }^{1,2}$. Ciliopathies can affect a range of organ systems and tissues, including the kidneys, liver, eyes, testes, brain and skeleton. Often, multiple organ manifestations occur in combination (e.g. Bardet-Biedl Syndrome affecting kidney, brain, eyes, gonads and digit number), but depending on the affected locus, only certain combinations of phenotypes may co-occur $^{3,4}$.

Most genes affected by mutations in ciliopathy patients encode for structural components of the primary cilium $^{1-3}$. Some protein groups co-localize to ciliary subcompartments, and are associated with common clinical features. Examples are the Bardet-Biedl related BBSome components at the base of cilia ${ }^{5}$, proteins in the proximal cilium that are mainly linked to nephronophthisis ${ }^{6}$, or components of the axonemal intraflagellar transport (IFT) machinery linked to skeletal ciliopathies (SC) such as Jeune's asphyxiating thoracic dysplasia (JATD), Mainzer-Saldino syndrome (MZSDS) and Sensenbrenner syndrome or Cranioectodermal dysplasia (CED) ${ }^{4,7,8}$. However, many ciliopathy proteins don't adhere to these categories. For example, mutations of basal body genes (CEP120) were identified in SC patients ${ }^{4,9}$, and IFT components (IFT27, IFT74) can cause Bardet Biedl syndrome ${ }^{10,11}$. Thus, the molecular cause of the variability and clinical overlap of certain ciliopathy manifestations is elusive.

Despite recent advances in genetics that identified most genes affected in ciliopathies, and studies of the ciliary proteome in cell culture models, the understanding of ciliary protein compositions and their function in each specific tissue remains incomplete ${ }^{12-14}$. Posttranslational modifications (PTMs) of axonemal tubulin by polyglycylation and -glutamylation contribute to the "tubulin code" that diversifies tubulin function and supports the structural integrity of cilia ${ }^{15-17}$. In vitro studies have shown that ciliary polyglutamylation by TTLL5 and TTLL6 depends on the Joubert syndrome associated proteins ARL13B and CEP41 ${ }^{18,19}$, but if tubulin PTMs are defective in other ciliopathies or contribute to the phenotypic pleiotropy remains unknown.

One subgroup of ciliopathies affects patients with skeletal deformities ${ }^{4,9}$. Typical phenotypes of SCs are shortened ribs resulting in a narrow thorax, truncated limbs (micromelia), shortened fingers (brachydactyly), and multiple digits (polydactyly). In some cases, the kidneys (nephronophthisis or cystic kidneys), eyes (retinopathy), liver function (liver fibrosis, liver cysts), or left-right axis formation (situs inversus) can be affected.

The Hedgehog $(\mathrm{Hh})$ signaling pathway is one of many cellular signals that is frequently found to be disrupted by defective ciliary function ${ }^{20-32}$. The ciliary $\mathrm{Hh}$ pathway is required for chondrocyte proliferation, differentiation, and limb patterning ${ }^{33,34,35,36}$. Two of the best characterized SC genes are ift80 $0^{37-39}$ (mutated in SRPS III and IV and JATD) and ift172 ${ }^{40-42}$ (mutated in JATD and MZSDS). Both are IFT-B subcomplex proteins ${ }^{43}$ and although genetic interactions were described, they do not interact physically with each other ${ }^{40,43,44}$. In model organisms, biallelic loss-of-function mutations result in shortened, missing or fewer cilia. Morphant and mutant zebrafish larvae have pronephric glomerular cysts, retinal degeneration, pericardial edema, slightly smaller eyes, and a curved tail ${ }^{39,40,45}$. In addition, ift172 morphants have otolith defects, hydrocephalus and craniofacial cartilage defects ${ }^{40}$. In mice, null 
alleles of ift80 or ift172 are prenatally lethal ${ }^{20,24}$, while mice with hypomorphic alleles have a low survival rate and suffer from shortening of long bones, a narrow thorax and polydactyly ${ }^{24,46,47}$. Chondrocyte specific loss-of-function of either gene in conditional knockout mice results in severely shortened $\operatorname{limbs}^{48,49}$. Conditional Col2 $\alpha 1$; IFT80 $0^{\mathrm{fl} / \mathrm{ll}}$ mice revealed that chondrocytes are disorganized, the growth plate is smaller, and articular cartilage is thickened ${ }^{48,50}$. Loss of ift80 or ift172 reduces the expression of Hedgehog target genes, such as patched1 or gli1 ${ }^{24,47}$, in line with the hypothesis that $1 \mathrm{ft} 80$ acts downstream of Smoothened as its agonist SAG and the second messenger Gli2 can rescue the loss of Ift $80^{48}$. Embryonic renal dysplasia has been observed in mice with hypomorphic alleles of ift172, but no studies have addressed their role in kidney function.

Mutations in at least 27 known disease genes currently account for most genetic diagnoses in cases of $\mathrm{SC}^{4}$. However, because SC is a rare genetic disorder, the known causal genes might not cover the complete spectrum of genes relevant to the pathophysiology of SC. A more complete understanding of the molecular network of SC proteins is needed to shed light on its pathogenesis and offer potential therapeutic options.

Here we describe two novel models for chondrodysplasia using CRISPR/Cas9 mediated editing of ift80 and ift172 in Xenopus. CRISPR targeted froglets developed severe limb defects during metamorphosis with shortened limbs due to chondrocyte differentiation defects, syn- and polydactyly, and polycystic kidneys, phenocopying major clinical features of ciliary chondrodysplasia. Impaired ciliogenesis was detected in multiciliated cells and resulted in defective fluid excretion during tadpole stages. Using a data-mining approach, we identified candidate genes with similar molecular properties to the established disease genes. Among these, only ttc30a targeting mimicked the full spectrum of chondrodysplasia phenotypes, i.e. impaired ciliogenesis, fluid retention in tadpoles, polycystic kidneys, and strongly affected limb development, including polydactyly in froglets. Thus, Ttc30a acts in ciliary signaling disrupted in chondrodysplasia and cystic kidney disease. Enhanced expression in chondrocytes and osteocytes of Ttc30a1 and Ttc30b suggest an evolutionarily conserved role in mammals. Together, our findings identify TTC30A/B as a critical node in the pathogenesis network of ciliary chondrodysplasia with polycystic kidney disease.

\section{Results:}

\section{CRISPR/Cas9 targeting of ift80 and ift172 leads to chondrodysplasia and cystic kidneys in}

\section{Xenopus tropicalis.}

We chose Xenopus to establish a model for ciliary chondrodysplasia, because it is an aquatic organism that allows for analysis of limb development, has a high sequence conservation to humans, is an established model in cilia research, and provides good experimental access ${ }^{50-54}$. We targeted two well characterized SC genes (ift80 and ift172) for loss-of-function analysis using CRISPR/Cas9 based genome editing. Indel frequency and knockout scores were used to identify highly effective sgRNAs against both targets in $X$. tropicalis embryos (Supplement Fig. 1 a-c). Injection of F0 embryos with Cas9/sgRNA ribonucleoprotein (RNP) resulted in mosaic gene disruption, and has previously been shown to elicit specific phenotypes at high frequency and penetrance ${ }^{55-60}$. 
Phenotypic analysis of 3-4 day old embryos (stage 45) injected at the one-cell stage showed prominent generalized edema in both ift80 and ift172 targeted embryos. Importantly, this phenotype was rescued by co-injecting ift80 or ift172 mRNA, demonstrating the specificity of the sgRNA mediated loss-offunction (Fig. $1 \mathrm{a}, \mathrm{b}$ ). Morpholino oligonucleotide knockdown in X. tropicalis and CRISPR/Cas9 targeting elicited the same phenotype in $X$. laevis confirming the specificity of the phenotype in a separate species (Supplementary Fig. 1 d-j).

Embryos targeted for ift80 or ift172 disruption sometimes had smaller heads and narrower eye width which often occurred in combination with head edema (Fig. 1 a; Supplementary Fig. 1d, e). Craniofacial defects also appeared at high rates in X. laevis CRISPR/Cas9 targeted embryos (Supplementary Fig. $1 \mathrm{~g}, \mathrm{~h})$.

The most prominent phenotypes in chondrodysplasia patients are malformations of the axial skeleton and limbs, i.e. shortened ribs, long bone dysplasia, and polydactyly ${ }^{4}$. Unilateral CRISPR-targeting of F0 animals has been shown to be effective to disrupt genes essential for post-metamorphic limb development in Xenopus ${ }^{61-63}$. To test if Ift80 and Ift172 act in limb development in Xenopus we unilaterally injected two-cell stage embryos, which limits gene editing to only one half of the body while retaining the unaffected half as an internal control. We targeted s/c45a2 as an additional negative control, which results in a loss of pigmentation that can be readily observed at earliest stages and controls for the correct delivery and function of the Cas9/RNP-endonuclease ${ }^{59,64}$. Apart from the expected loss of skin pigmentation, metamorphic froglets were phenotypically normal, demonstrating that sgRNA/Cas9 injection per se did not have an impact on development (Fig. 1 c, Supplementary Fig. $4 \mathrm{j})$.

In contrast to controls, froglets that were CRISPR-edited at the ift80 or ift172 locus showed severe limb defects. Both fore- and hindlimbs were substantially shorter than in uninjected control or s/c45a2 targeted froglets (Fig $1 \mathrm{c}, \mathrm{d}$, e, green and red arrowheads). In addition, limb diameters were clearly thickened compared to controls.

In most animals the fingers and toes were shortened and less developed, sometimes not even identifiable (Fig. $1 \mathrm{~d}, \mathrm{e}$ ). The number and size of clawed digits of the hindlimbs varied substantially from wildtypes, in particular in ift80 targeted froglets. $X$. tropicalis usually possess six digits at the hindlimb, four of which form claws ${ }^{65}$. In ift80 targeted froglets, the number of clawed toes were consistently either reduced to less than three (syndactyly) or increased to six or seven (polydactyly) (Fig. $1 \mathrm{~d}$ ). In conclusion, this phenotype is highly reminiscent of the polydactyly phenotype observed in mouse models and human patients $24,37,40,49,66$.

In order to explore the phenotypic changes in more detail, we conducted microCT scans of mosaic mutant froglets. There was no difference between injected and uninjected sides of the control s/c45a2 targeted froglets, the length measurements were much less variable than in ift80 and ift172 (Fig. $1 \mathrm{c}$ e). Despite unilateral RNP injections, we observed bilateral limb malformation in some animals, suggesting that in these cases, the first cell cleavage had not fully separated at the time of injection, allowing for gene editing to occur across the midline. Comparing limbs of ift80 and ift172 disrupted froglets on the injected versus the uninjected side, we detected a strong shortening of forelimbs and a less pronounced length difference of the hindlimbs (Fig. 1 c-e). lodine based contrast enhancement 
allowed us to distinguish muscle, cartilage, and other internal organs based on density differences. We found that the shortened, not yet ossified limbs consisted mainly of cartilage accumulations (Fig $1 \mathrm{~d}$, e, green arrowheads), consistent with the phenotype in humans and mouse models ${ }^{48,50,67}$. Histological sections confirmed that the resting and proliferation zones of cartilage dominated clearly in ift80 and ift172 targeted animals at the expense of the hypertrophic and ossification zones visible in s/c45a2 targeted control animals (Fig. $1 \mathrm{c}-\mathrm{e}$ ). In general, the chondrocytes were less well organized in ift80 and ift172 depleted cartilage than in limbs of controls. Similar phenotypes have been described for conditional ift80 knockout mice and other chondrodysplasia models ${ }^{24,26,68-70}$.

Contrast enhanced microCT was also able to resolve the structure of the mesonephric kidneys in the post-metamorphic froglets at high resolution and revealed polycystic kidneys in most of the mutant animals (Fig. 2 a-c). Quantification and volumetric analysis showed both ift80 and ift172 disruption resulted in a significantly higher number of cysts $(>0.2 \mathrm{~mm})$ in comparison to slc45a2 controls, particularly on the injected side (Fig. 2 d). The occurrence of cysts on the uninjected side in ift80 mosaic mutants likely reflects the incomplete segregation of gene editing to one half, as observed for limb measurements. The total cyst volume was larger on the injected side of ift80 but not significantly changed for ift172 edited froglets (Fig. 2 e). The volume of non-cystic renal parenchyma was not significantly different (Fig. 2 f). To our knowledge, this is the first report of polycystic mesonephric kidneys in Xenopus.

We conclude that froglets with mutations in ift80 and ift172 displayed shortened extremities with polydactyly and cystic kidney disease, confirming that chondrodysplasia can be modelled in Xenopus. This supplements the available mouse and zebrafish lines, and offers new opportunities to analyze chondrodysplasia genes and their prospective functions $\mathrm{s}^{49,50,67,71}$.

\section{Ift80 and ift172 targeting affects multiciliated cells resulting in pronephric excretion defects.}

Next, we explored the effect of ift80 and ift172 disruption on a cellular level. The most prominent phenotype of ift80 and ift172 mutant tadpoles was generalized edema. Suggesting a deficiency in water or ion homeostasis due to pronephric, lymphatic, vascular, or cardiac insufficiency. In situ hybridization of established marker genes (aplnr, vasculature; $n k x 2.5$, heart; prox1, lymphatic system) did not reveal an obvious developmental disruption of any of these organ systems in Xenopus embryos targeted for ift80 and ift172 disruption (Supplementary Fig. 2 c, d ). Given the prominent renal phenotype in froglets, the edema phenotype in embryos, and because Jeune syndrome patients and mouse models frequently suffer from kidney disease, we focused on the pronephros of $X$. tropicalis embryos ${ }^{47}$. We did not find structural tubular defects when staining for the tubular epithelium (Tomato lectin; Supplementary Fig. 2 e, f) nor were the expression of segment-specific marker genes ( $n k c c 2$, distal tubule; sglt1 proximal tubule) affected (Supplementary Fig. 2 c, d) suggesting a normal differentiation of tubular tissue.

Expression analysis of ift80 and ift172 by in situ hybridization revealed both genes to be prominently expressed in the nephrostomes (Figure $2 \mathrm{~g}$ ). These represent the most proximal segments of the pronephric tubule that contain densely ciliated cells which facilitate uptake of coelomic fluid into the pronephric tubule (Figure $2 \mathrm{~h}$ ). To test whether impaired fluid uptake into the tubule could explain the observed edema phenotype in ift80 and ift172 bilaterally disrupted embryos, we employed a dextran 
excretion assay. Embryos were injected with sgRNA/Cas9 at the two- to four-cell stage, and fluorescently labeled dextran was injected into the coelom at stage 39-40 when the pronephros is fully functional. Time-lapse movies of untreated or s/c45a2 sgRNA injected (negative control) embryos showed rapid uptake and acceleration of dextran through the pronephric tubule and excretion at the cloaca on both sides in $84,6 \%$ of embryos (Fig. 2 j, k). In contrast, entry into the pronephric tubule was observed in only $12,5 \%$ and $13,8 \%$ of embryos within 3 min on the ift 80 and ift172 targeted side (Fig. 2 $\mathrm{j}, \mathrm{k}$ ), respectively. Dextran uptake was comparable to wildtype and much faster on the non-targeted side of unilaterally gene edited embryos. Normal excretion on the uninjected side could also be indirectly observed in the time-lapse movies where dextran entered the medium of ift80 and ift172 targeted embryos (Supplementary Movie 1).

Motile cilia in the nephrostomes actively propel fluid into the renal tubules. Thus, structural defects of ciliated cells, which has been described for ift80 and ift172 mutant zebrafish, may explain the observed loss of excretion in CRISPR targeted tadpoles ${ }^{45,71}$. To analyze the role of ift80 and ift172 in motile cilia, we turned to the epidermal multiciliated cells, a well established and accessible model for ciliogenesis. Centrin-RFP mRNA was used as an injection marker to identify ciliated cells that received the CRISPR RNPs and label basal bodies of ciliated cells. The mosaic distribution allowed us to analyze CRISPR targeted and wildtype cells in the same images. Cilia appeared to be less and shorter in length which was confirmed by quantification of cilia in ift80 and ift172 knockout cells. No such difference was observed in slc45a2-targeted controls (Fig. $2 \mathrm{l}, \mathrm{m}$ ). In contrast, basal body number and apical docking was not altered in crispant MCCs (multiciliated cells) when compared to controls (Supplement Fig. 2 g). Thus, the ciliogenesis defect is consistent with findings in other model organisms ${ }^{45,71}$ and a likely cause for impaired tubular uptake.

To gain more detailed insights into the molecular signals affected by a loss of ift80 or ift172, we investigated the transcriptional profile of embryos targeted for ift80 and ift172. Because embryos were in vitro fertilized and developed synchronously, stage dependent variability was minimized between control and CRISPR targeted embryos. We isolated DNA and confirmed a high indel frequency in the same samples subjected to RNA-Seq analysis at stage 22 (neurula) (Suppl. Figure $3 \mathrm{a}, \mathrm{b}$ ). The top most downregulated genes were ift80 and ift172, respectively (Fig. 3 a, Suppl. Fig. 3 c, d, and Suppl. Tables 1 and 2), confirming the specificity of our targeting strategy and a direct effect on mRNA levels likely due to nonsense-mediated decay. In each condition, we found 15 (ift80) and 5 (172) genes to be differentially regulated (Suppl. Fig. $3 \mathrm{c}$, d). We found three genes (abcb1 and two uncharacterized transcripts) to be significantly upregulated in both conditions (Fig. 3 a). The ATP-dependent multi-drug resistance transporter Abcb1 is transcriptionally regulated by Gli1 and $\beta$-catenin ${ }^{72,73}$. Interestingly, additional five genes that only reached significance in one condition were also associated with Hedgehog-signaling (ccng1, ulk1, riok3, olfm4, col1a1) and four with Wnt-signaling (c3ar1, col1a1, olfm4 and ulk1) (Figure $3 a)^{72,74-82}$. Thus, we detect Hedgehog- and Wnt-dependent gene regulation to be altered upon depletion of ift80 and ift172 in Xenopus embryos.

mRNA expression analysis in wildtype embryos of differentially regulated genes identified by RNA-Seq found an overlapping expression with chondrodysplasia genes in neuronal tissue and neural crest. This was particularly true for ccng1, ulk1 and riok3 (Supplement Fig. 3 e). However, we did not find a change 
in the Sonic Hedgehog marker $n k x 2.2$ by in situ after unilateral ift80 or ift172 targeting, suggesting that the differences in mRNA abundance are below the detection level of in situ hybridization (Suppl. Fig. 3 $f, g)$.

\section{Data mining identifies skeletal ciliopathy associated genes}

Next, we aimed to identify novel proteins related to the pathogenesis of SC. We reasoned that proteins with similar molecular properties, such as subcellular localization, common protein interactors, or differential regulation in loss-of-function models, could be unrecognized components of the pathogenetic molecular mechanism. Therefore, we assembled lists of genes from 90 published genome-wide screens or unbiased analyses ("resource lists") (Suppl. Table 3) based on literature curation. Because these lists were obtained from screening experiments generated across various species, all gene identifiers were matched to the closest human ortholog. We identified 16 resource lists to be significantly enriched for genetically validated chondrodysplasia genes in comparison to 10 million random draws of an equal number of query genes (Figure $3 \mathrm{~b}$ ). Within these 16 lists, 65 unique genes not yet linked to chondrodysplasia were found to be significantly enriched (Suppl. Table 4). Among these potential candidate genes were three that have been identified as bona fide chondrodysplasia genes in the meantime (i.e. ift122, ift43 and traf3ip $1^{70,83,84}$ ). Ift22 was described to be mutated in a short rib- polydactyly syndrome (SRPS) type IV patient, ift43 and traf3ip1 in SRPS type II patients and traf3ip1 additionally in a case of JATD ${ }^{70,83,84}$. This confirmed that our approach was able to predict valid genetic associations.

Given that the system worked well with chondrodysplasia genes, we used all gene sets annotated in phenotypic series in OMIM as input. A significant number of enriched lists could be identified for 13 disease entities, many of which were ciliopathies (e.g. Meckel syndrome, nephronophthisis, primary ciliary dyskinesia; Suppl. Table 6).

Among the 65 potential chondrodysplasia related candidate genes, we focused on genes that have not been linked to any ciliopathies nor characterized in a knockout mouse model. We focused on the top four genes with the lowest p-value, that were targetable in X. tropicalis (C11ORF74, CEP192, CFAP20 and TTC3OB) for functional analysis (Fig. $3 \mathrm{c}-\mathrm{g}$ ). All proteins encoded by these four candidate genes had previously been associated with cilia. CFAP20 (cilia and flagella associated protein 20) regulates cilia length and morphology ${ }^{85,86}$. Entering of the BBSome into the cilium via interaction with the IFT-A complex is controlled by C11ORF74 ${ }^{87}$. CEP192 (centrosomal protein of $192 \mathrm{kDa}$ ) is required for mitotic centrosome and spindle assembly ${ }^{88,89}$. TTC30B (tetratricopeptide repeat domain 30b, also known as IFT70) is essential for polyglutamylation and polyglycylation of axonemal tubulin and is part of the IFT$B$ complex ${ }^{90-92}$. ТTС30B has one closely related homolog in humans (TTC30A) and three in mice (Ttc30a1, Ttc30a2, Ttc30b), but only one ortholog in $X$. tropicalis and Danio rerio (ttc30a/fleer) (Fig. 4 a) $)^{91}$. 
We designed sgRNAs targeting all Xenopus orthologs of the four candidates for CRISPR/Cas9 mediated loss-of-function experiments (Fig. 4 b, c; Supplement Fig. 4 a-d). Interestingly, cfap20 and ttc30a crispants formed edema suggestive of embryonic renal excretion defects. This effect was specific as co-injection with corresponding mRNAs significantly rescued the edema phenotype (Figure $4 \mathrm{c}$, Suppl. Fig. 4 c, d).

In order to evaluate a potential effect on limb development, we raised cfap20, ttc30a, c11orf74, and cep192 CRISPR targeted tadpoles to metamorphosis. cep192 LOF resulted in a slowed development compared to controls and therefore retained a small stature, while c11orf74 and cfap20 crispants did not show any obvious phenotypes and limb formation was normal in all three conditions (Suppl. Fig. 4 j, k). Froglets with ttc30a mutations, however, developed severe limb defects (Fig. 4 d, green arrowheads). Extremities were generally shorter and thickened, digits were shortened and demonstrated a polydactyly phenotype (Fig. 4 d; Suppl. Fig. 4 h). MicroCT scans found large depositions of cartilage. Histology revealed a chondrocyte differentiation defect, as previously observed for ift80 and ift172 targeted animals. Ttc30a targeted embryos had reduced fore- and hindlimb length (Fig. 4 d) and analysis of the kidneys revealed a significantly higher number of cysts and in the total cyst volume (normalized to kidney size) on the injected side of the froglets (Fig. 4 e). Excretion assays confirmed that renal tubular uptake of dextran injection into the coelom was drastically reduced (Fig. 5 $a, b)$, and epidermal cilia were shorter or absent in embryos injected with sgRNA and RNPs targeting ttc30a (Fig. 5 c, d). In conclusion, CRISPR targeting ttc30a in Xenopus replicated all phenotypes observed when targeting the chondrodysplasia genes ift80 and ift172.

To elucidate the expression pattern of mammalian orthologs of $t t c 30 a$, we analysed publicly available single cell sequencing data of E13.5 mouse limb buds (Suppl. Fig. 5 ) $^{93}$. We found that among the three orthologs, Ttc30b and to a lesser degree Ttc30a1 were most prominently expressed in cartilage, bone and growth plate clusters (Fig. 5 e-g). The distribution of cell proportions expressing Ttc $30 \mathrm{~b}$ was similar to that of Ift80 and Ift172 (Fig. $5 \mathrm{~g}, \mathrm{~h}$ ). Thus, the expression pattern of Ttc30b and Ttc30a1 suggest a potential role in cartilage formation of mammals. We screened for TTC30A/B mutations in a cohort of 24 unresolved severe ciliary chondrodysplasia cases by exome sequencing, but no likely pathogenic variants were identified. Screening of whole exome sequencing data in a worldwide cohort of more than 500 patients with nephronophthisis related ciliopathies and cystic kidney disease also did not reveal potentially causative recessive mutations in either TTC30A or TTC30B.

\section{Discussion:}

Skeletal ciliopathies are caused by pathogenic mutations in genes that encode ciliary proteins. Thus, Mendelian genetics put the spotlight on the primary cilium as a central organelle in the pathophysiology of chondrodysplasias. This led to the insight that several clinically distinct disease entities may have a common molecular origin and are now recognized as an united disease group ${ }^{4}$. Today, a causative mutation can be found in almost all patients with SC, both in frequently affected genes and rare loci, which may only occur in a few patients worldwide. 
Despite these enormous achievements, a full understanding of all disease-associated proteins, their functional relationships and their molecular functions is incomplete. Therefore, novel in vivo models can help to assign tissue specific functions of ciliary proteins molecularly characterized in vitro. The tetrapod Xenopus tropicalis is both easy to manipulate but not burdened by genome duplications that complicate genetic analysis in other aquatic models ${ }^{94,95}$. Thus, our work demonstrates that disruption of Xenopus orthologs of human SC disease genes elicit phenotypes highly consistent with the clinical presentation in patients.

Early limb bud development in Xenopus is very similar to that of other tetrapods on a molecular level ${ }^{52}$. As in humans, mouse, and chicken, cilia and cilia-related signaling pathways (Hedgehog ( $\mathrm{Hh}$ ) and Wnt) appear to play an essential role in shaping cartilage and future bone size also in Xenopus. Most SCgenes have been described to influence $\mathrm{Hh}$ signaling ${ }^{4}$. Among the Hh-ligands, Sonic hedgehog (Shh) defines the anterior-posterior axis of the limb and therefore the number of digits ${ }^{96}$, while Indian hedgehog (Ihh) forms a gradient in the growth plate which controls chondrocyte proliferation versus maturation and therefore bone lengthening ${ }^{35,97}$. As we observed phenotypes consistent with defects in Shh (polydactyly) and Ihh (brachydactyly), both pathways may be affected in our model ${ }^{35,36,98}$. Interestingly, knockout mice of a cilia-dependent regulator of both ligands, gpr161, shows the same phenotype, which is however limited to the forelimbs ${ }^{99}$.

Wnt signaling has multiple functions during limb development, but it is not known to what degree Wnt signaling is affected in SC patients ${ }^{100}$. It has been described that $\mathrm{Wnt} / \beta$-Catenin signaling, in concert with Shh, acts in chondrocyte differentiation and is balanced by ift $80^{48}$. Some SC-genes are linked to Wnt/PCP (planar cell polarity) signaling, which affects the organization of chondrocytes ${ }^{26,27,101,102}$. Indeed, polysyndactyly in combination with brachydactyly, that we also observe in our SC-models, is typical for a loss of HOXD13, a gene that regulates Shh as well as WNT5a (Wnt/PCP) during limb development ${ }^{101,103,104}$. A potential involvement of both, Wnt and Hh pathways may explain why we detected changes in transcripts that impact with both signaling pathways during embryogenesis (Fig. 3 a).

A trove of existing datasets, proteomic and functional screens have contributed to a better understanding of the protein composition of cilia ${ }^{105,106}$. Our data-mining approach integrated these high quality datasets to provide underappreciated links between ciliary proteins and human disease, and ciliopathy subtypes in particular. We created a web application to make the resource lists used in our in silico screen accessible and searchable (http://dormantdata.org). Adding further datasets and using novel machine learning algorithms may further enhance the power of a data-driven discovery process. Such refinements could make this approach be applicable to other non-ciliary diseases in the future. By combining the in silico screen with in vivo modelling, we identified Xenopus Ttc30a as a protein involved in chondrocyte differentiation and renal function. It is likely that not all SC relevant components are known. Potentially deleterious variants may be too rare to be identified by Mendelian genetics because the natural mutational load does not reach saturation for all possible pathogenic mutations to occur, or lead to early embryonic lethality. In addition, essential proteins may be protected by genetic compensation of functionally redundant genes ${ }^{107,108}$. The human $T T C 30 A / B$ locus may represent a 
critical site that is protected by early and reoccurring duplication events in the tetrapod phylogeny that increasingly relied on limbs for locomotion and dexterity (Fig. 4 a). TTC30A/B are highly conserved in eukaryotes. The amino acid sequence between the green algae Chlamydomonas rheinhardtii and human TTC30A is $73 \%$ identical $^{90}$. In zebrafish, the fleer (ttc30a) mutant shows a general ciliopathy phenotype, including pronephric cysts, dorso-ventral body axis curvature, laterality defects, hydrocephalus, and retinal degeneration ${ }^{91,109}$. Cilia are either shorter or absent (pronephros, Kupffer's vesicle and olfactory placode) and lack parts of the microtubule-duplets of the axoneme. Both, in vitro and in vivo experiments identified ttc30a as a peripheral protein of the IFT-B1 subcomplex ${ }^{110,111}$, where it links the ift88/ift52-complex with Kif-proteins (Kif17) ${ }^{112}$. Further, ttc30a is necessary for polyglutamylation and polyglycylation of tubulin by regulating the ciliary activity of ttll3/6 (Tubulin Tyrosine Ligase Like 3/6), and ccp5 (cytoplasmic carboxypeptide 5) ${ }^{15,92}$. Both posttranslational modifications stabilize tubulin complexes and are necessary for cilia motility ${ }^{92}$. Interestingly polyglutamylation regulates velocity of the Kinesin-2 complex and therefore the uptake of Hedgehog components (Smo, Gli3) after signaling activation, and has recently been found to be dependent of the Joubert syndrome associated proteins ARL13B and CEP41 ${ }^{18,19,113}$. It is not known whether tubulin polyglutamylation is affected in other ciliopathies. Intriguingly, the ciliary localization of PKD2 was found to depend on tubulin glutamylation, suggesting a role in renal cystogenesis ${ }^{18,114}$.

Like glutamylation, tubulin glycylation appears to be present only in primary cilia of certain cell types. Glycylation was detected in MDCK cells, but absent in IMCDs and RPEs ${ }^{16}$. Only a subset of cilia in the spinal cord and pronephros are glycylated, but not Kupffer's vesicle cilia of zebrafish ${ }^{92}$. Thus, tubulin amino acid PTMs not only constitute a part of the "tubulin code" that diversifies ciliary functions, but may contribute to the pleiotropic phenotypes of ciliopathies ${ }^{114}$.

The molecular function of TTC30A/B in ciliogenesis is well established, but has not been associated with skeletal defects. In human tissue, TTC30A and TTC30B are strongly expressed in ciliated tissues, such as airway epithelial cells, ciliated cells of the ductuli efferentes in the epididymis, and the spermatozoa progenitor tissue of the seminiferous ducts ${ }^{115}$. Consistent with our own analysis, single cell RNA-Seq data of embryonic mice revealed an enhanced expression of ttc30a1, ttc30a2 and ttc30b in osteoblasts and chondrocytes (Fig. 5) ${ }^{116}$. A rare missense variant in TTC30B (rc.1157C > T, p.A375V) has recently been described in a large Chinese family with polysyndactyly, suggesting an autosomal dominant mode of inheritance ${ }^{117}$. However, no other unifying clinical phenotypes were observed in the 27 affected family members. In vitro analysis suggested that downregulation of TTC3OB had an inhibitory effect on Shh-pathway stimulation, consistent with our findings that Ttc30a has a role in limb patterning. Hedgehog signalling does not influence cystogenesis in autosomal polycystic kidney disease (ADPKD), suggesting that other ciliary mechanisms are more likely to be at play ${ }^{118}$. Further investigation will need to elucidate, which signals are disrupted by loss of Ttc30a in renal cyst formation and to what degree polyglutamylation and -glycylation are more widely implicated in nephronophthisis and other ciliopathies.

\section{Material and Methods:}




\section{Animal maintenance and handling}

Adult animals were kept according to the German and Swiss law for care and handling of research animals. Husbandry and treatment were approved by the local authorities (Regierungspräsidium Freiburg and Veterinäramt Zürich).

Xenopus embryos were kept in 0.3x Marc's Modified Ringer (MMR, HEPES (free acid) $5 \mathrm{mM}$, EDT 0.1 $\mathrm{mM}, \mathrm{NaCl} 100 \mathrm{mM}, \mathrm{KCl} 2 \mathrm{mM}, \mathrm{MgCl}_{2} 1 \mathrm{mM}, \mathrm{CaCl}_{2} 2 \mathrm{mM}$ ) with $0.05 \mathrm{mg} / \mathrm{ml}$ Gentamycin. Premetamorphic tadpoles were raised on Sea Micron (Heinsberg, Germany) at room temperature with daily buffer/water exchange. After approximately 4 weeks the food was changed to Sera Vipagran Baby (Heinsberg, Germany) and after approx. 8 weeks crushed adult frog food (Shrimp Sticks) was fed. Staging was performed according to Nieuwkoop and Faber ${ }^{119}$.

\section{Plasmids, mRNAs, sgRNAs and MOs}

For gene expression analysis of SC reference and candidate genes, $X$. laevis gene variants were either cloned into pGEM-T (ilft80) or pBluescript (iff172) or a PCR-product was used as template for antisense probe synthesis (ttc30a) (X. laevis) after addition of the T7 promoter site to the reverse primer.

The constructs pax2-a and pax6 (X. laevis) were previously cloned in our lab ${ }^{120}$. prox1, aplnr, nkx2.5, gli2, patched1, cacgn1, ccng1, cfh, olfm4, ulk1 and riok3 (X. tropicalis) were cloned into pGEM-T. Further constructs were kindly provided by M. Blum (snail ${ }^{121}$, twist1. $S^{122}$ and sox9), J.B. Wallingford $(n k x 2.2)^{123}, \mathrm{~N}$. Ueno $(n k c c 2)$ and O. Wessely $(s g l t 1 k)^{124}$, all $X$. laevis. For antisense probe preparation, plasmids were linearized and transcribed with T3, T7, or SP6 polymerase (Roche, Basel, Switzerland). The coding sequence of $X$. tropicalis ift80, ift172, and ttc30a were cloned into VF106 for rescue experiments. Constructs were linearized with Sall (ift172, X. laevis and ttc30a, X. tropicalis) or Ascl (ift80, $X$. laevis) for mRNA synthesis. mRNA was made with mMESSAGE mMACHINE T7 Transcription Kit (Invitrogen, AM1344) and cleaned up with RNeasy mini kit (Qiagen, Cat No./ID: 74104).

Short guide RNAs were designed with chopchop and CRISPRscan (https://chopchop.cbu.uib.no; https://www.crisprscan.org) for CRISPR/Cas9 targeting ${ }^{125,126}$. The PCR-based method was used for sgRNA synthesis described by Nakayama et al. $2013^{127}$. The PCR-product was amplified with the KOD polymerase (Novagen; 71086) and purified with the QIAquick PCR Purification Kit (Qiagen; Cat No./ID: 28104). sgRNAs were synthesized overnight with the MEGAscript T7 Transcription Kit (Invitrogen; AM1334). Afterwards sgRNAs were cleaned-up with the mirVana miRNA Isolation Kit (Ambion; AM1560). Several sgRNAs were tested for each gene and the most efficient sgRNA was used for experiments, as determined by ICE or HRMA analysis. For $X$. laevis experiments, previously published tyrosinase sgRNAs was used ${ }^{53}$.

To verify functionality of ift80 and ift172 sgRNA in experiments where unilateral injections for limb analysis were performed, high dose injections at 1-cell stages were done in parallel, and edema formation was confirmed to occur at stages $42-45$ (Suppl. Fig. 1n, 4g).

Morpholino oligonucleotides for $X$. laevis Ift80, Ift172 and Ift52 were designed by and ordered from genetools. Additionally the Standard Control MO from genetools was used for injected controls. Sequences of primer and morpholino oligonucleotides are given in Supp. Table 5. 


\section{Microinjections}

Embryos were obtained by in vitro fertilization ( $X$. laevis and tropicalis) or natural mating (X. tropicalis). For injections, embryos were transferred to $3 \%$ Ficoll with $0.1 \%$ BSA (X. tropicalis) or $2 \%$ Ficoll $(X$. laevis) dissolved in 0.3x MMR. Depending on the experiment embryos were injected the 1-cell stage (phenotype analysis, genotyping and RNA-Seq) or unilaterally at 2-4 cell stage (all other experiments). The injected volume was $10 \mathrm{nl}$ in $X$. laevis and $5 \mathrm{nl}$ in $X$. tropicalis. In all experiments a lineage tracer (2ng Fluorescein-Dextran 70kMW - Invitrogen; D1823) was co-injected, and embryos sorted according to the injected side at neurula stage. For experiments on epidermal cilia, 0.2ng Centrin-mRFP (kindly provided by J.B. Wallingford) $)^{128}$ was co-injected.

For CRISPR/Cas9 experiments, $0.175 \mathrm{ng}$ sgRNA was injected in combination with $0.6 \mathrm{ng}$ Cas9 protein with NLS (PNABio; CP01). As a control, sgRNA against s/c45a2 (X. tropicalis) or tyrosinase (X. laevis) was used. Rescue experiments were performed with $0.1 \mathrm{ng}$ ift80, $0.15 \mathrm{ng}$ ifft172 or $0.2 \mathrm{ng}$ ttc30a $X$. tropicalis mRNA. For raising crispants $0.015-0.02 \mathrm{ng}$ ift80 or ift172-sgRNAs, $0.02-0.05 \mathrm{ng}$, and ttc30a sgRNA was injected at the 2-cell stage. The same amount was used for controls.

In X. laevis 3.6 - 14.5ng ift80 TB MO; 3.6 - 7.25ng ift172 TB MO as well as ift52 TB MO were injected in knockdown experiments. In $X$. tropicalis half of the amount was used. The same concentration of a Standard Control MO was injected as control.

\section{Genotyping}

sgRNA efficiency was tested by genotyping embryos after 1 to 3 days. Five injected individuals per sgRNA were lysed and DNA extracted with the DNeasy Blood and Tissue Kit (Qiagen; Cat No./ID 69504). Genotyping was performed with high resolution melting analysis ${ }^{129}$ using the MeltDoctor ${ }^{\mathrm{TM}}$ HRM Master Mix (Applied Biosytems) on a Roche LightCycler 480. The DNA-product of the analysis was purified (QIAquick PCR Purification Kit) and Sanger sequenced. Sequencing results were analyzed with ApE (A plasmid Editor v2.0.50b3) and the ICE-tool from Synthego (https://ice.synthego.com/\#/).

\section{Transcriptomic analysis}

Three independent replicates of ift 80 and ift 172 CRISPR/Cas 9 targeting experiments were analyzed by RNA-seq. One-cell stage embryos were injected, and Cas9 protein injections served as negative controls. Embryos were lysed at stage 22 and RNA isolated with the RNeasy mini kit (Qiagen, Cat No./ID: 74104). Afterwards, genomic DNA was extracted from the columns using 8mM NaOH on the filter to solve the DNA and EDTA $(0.57 \mathrm{mM})$ and HEPES $(426 \mathrm{mM})$ to stabilize it. HRMA and ICE analysis were performed to confirm successful indel formation. RNA was further purified with RNeasy Universal Plus kit from Qiagen (Cat No./ID: 73404) and Illumina sequenced by GATC (Germany). RNA-seq analysis was performed on the Galaxy platform ${ }^{130}$. Raw reads were trimmed (Trim Galore!) and aligned to the $X$. tropicalis genome version 9.1 using STAR ${ }^{131}$. Gene counts were calculated using featureCounts and differential expression was determined by DESeq2, taking batch effects of the replicates into account ${ }^{131,132}$. For single cell RNA analysis, disassociated limbs data for E15.5 time point from GEO accession number GSE142425 was analysed using R v4.0.2 and Seurat v3.1.5 ${ }^{133,134}$. Cells 
expressing less than 1200 or more than 4200 genes and a mitochondrial gene percentage of $>10 \%$ were excluded in QC leaving 2142 cells in total. Highly variable genes $(N=2500)$ were extracted from the normalized data, scaled and further used in downstream analysis. Data was clustered with $\mathrm{t}-$ distributed stochastic neighbor embedding (tSNE) using first 10 PCs for dimensionality. Cell types were assigned for 10 clusters (eliminating three clusters) using the markers reported in Kelly et al. ${ }^{93}$.

\section{Analysis of craniofacial defects}

For early phenotype analysis, embryos were fixed with MEMFA $(0.1 \mathrm{~mol} / \mathrm{L}$ MOPS (3morpholinopropane-1-sulfonic acid), $2 \mathrm{mmol} / \mathrm{L} \mathrm{EGTA}, 1 \mathrm{mmol} / \mathrm{L} \mathrm{MgSO}_{4}, 3.7 \%$ formaldehyde, $\mathrm{pH} 7.4$ ) at stage $42-45$ for approx. $2 \mathrm{~h}$ at room temperature and transferred to $1 \mathrm{x}$ phosphate-buffered saline (PBS) for imaging. Pictures were taken from the dorsal side of the embryo with a SteREO Discovery.V8 microscope and ZEN 2011 (blue edition; Zeiss, Oberkochen, Germany) software. The shortest distance between the eyes was measured using the line tool of imageJ 2.0.0. As analyzed embryos of different batches differed in age, the measured distances were normalized to the control embryos of the same batch.

\section{Whole mount in situ hybridization}

Whole mount in situ hybridization was performed as described by Sive et al. ${ }^{135}$. Embryos were fixed at desired stages with MEMFA for $1.5-2 \mathrm{~h}$ at room temperature. Antisense probes were detected with a phosphatase-conjugated secondary DIG-antibody (Roche, Basel, Switzerland). Stained embryos were bleached for 1-2h with $\mathrm{H}_{2} \mathrm{O}_{2}$ and methanol (1:2). Afterwards they were rehydrated with 1x PBS and imaged with a SteREO Discovery.V8 microscope and ZEN 2011 (blue edition) software.

\section{Immunostaining}

For immunostaining, embryos were fixed for $1-2 \mathrm{~h}$ in MEMFA. The staining was performed as described before $^{136}$. Cilia were detected with a monoclonal anti-acetylated tubulin antibody (Sigma, T7451) in combination with an anti-mouse secondary Alexa Fluor 488 antibody (Invitrogen, A-11001). Pictures were taken with a ZEISS LSM 510 DUO with inverted microscope Axiovert 200 and a $63 x$ LCI-Plan Neofluar objective and a Leica SP8 inverse FALCON and a 63x HC PL APO CS2 objective. Ciliation was determined in Centrin-RFP-positive cells and the area of their acetylated tubulin staining was measured on maximum intensity projections with ImageJ 2.0.0.

For pronephros analysis a fluorescein-labeled Lycopersicon esculentum lectin (1:100 dilution; Vector Laboratories) was used for visualization. A SteREO Discovery.V8 from Zeiss and Zen2011 Blue Edition was used for imaging. Pronephros size was measured with the line tool of ImageJ 2.0.0 and the ratio of injected versus uninjected side calculated ${ }^{6}$.

\section{Excretion assay}

Excretion assays were performed as described before ${ }^{137}$. Embryos were anesthetized with $0.2 \mathrm{mg} / \mathrm{ml}$ MS-222 around stage 38. Approximately 20nl of Fluorescein-labeled Dextran (70kMW - Invitrogen; D1823) was injected into the coelomic cavity around the heart. Uptake and excretion of Dextran was 
documented by performing time-lapse microscopy ( $2 \mathrm{fps}$ for a time-span of 4 minutes). Pictures were taken with the SPOT Insight FireWire system (Diagnostic Instruments) on a Leica MZ16 stereomicroscope and the SPOT advanced software. For visualization time-lapse movies were converted to AVI-movies of $40 \mathrm{fps}$ with imageJ 2.0.0.

\section{Analysis of CRISPR/Cas9 froglets}

Froglets for limb analysis were euthanized and fixed in MEMFA. lodine contrast enhancement was done by washing froglets in 1x PBS Buffer for 2 days, followed by dehydration to ethanol, and staining in $1 \%$ iodine solution (Sigma-Aldrich, 207772) in ethanol. The day before microCT scans, froglets were washed with $100 \%$ ethanol overnight. The animals were scanned with a Caliper Quantum Fx from PerkinElmer. To stabilize froglets in the microCT scanner, a piece of $1 \%$ agarose solved in water and dehydrated in $100 \%$ ethanol was used. MicroCT DICOM pictures were converted with Imaris File Converter 9.5.1 to imaris files. Imaris 9.5.1 was used to determine the volume of kidneys and the volume of kidney cysts. Length of limbs was measured with RadiAnt DICOM Viewer 5.5.1. To determine the total length of the hindlimb, length of femur and tibia/fibula was measured and summed. Length of forelimbs was determined by adding the length of humerus and ulna/radius.

\section{Histology}

lodine staining of froglets was removed with Thiosulfate as described in Hopkins et al. $2015^{138}$. Skin and muscles were removed from limbs and bone/cartilage washed with PBS. Preparations were decalcified with $20 \%$ EDTA - citric acid, pH 7.5 solution (BIOCYC, 400500201) for 3-5 days at room temperature. Limbs were dehydrated and embedded in paraffin. $5 \mu \mathrm{m}$ sections were made with the rotary microtome Microm HM355S. Sections were stained with Hematoxylin and Eosin following standard methods.

\section{In silico screen to identify novel SC-candiates}

For the in silico screen, we collected 90 publicly available datasets representing the results of various screens and unbiased large-scale experiments in various species (resource lists, Suppl Table 3). Gene names were mapped to the human orthologue (HGNC) using biomart and manually curated. Online Mendelian Inheritance in Man (OMIM) "phenotypic series" data was aggregated from the genemap.txt file downloaded on 26.01.2017 to obtain the 388 input lists of known and confirmed genetic diseases by matching phenotype descriptions to gene symbols.

First, each resource list was tested by Fisher's exact test, in which multiple testing was accounted for by use of a Bonferroni correction for the number or resource lists, to determine if the genes in the input list were enriched $(p<0.05 / 90)$ in a given resource list ${ }^{139}$. Genes on resource lists that showed significant enrichment of known monogenic genes on input lists were scored according to either their position in ranked resource lists $\left(\sigma_{\text {ranked }}=\frac{r^{r a n k}-1}{\sum \text { ranks }}\right)$ or by the total number of genes in the resource list $\left(\sigma_{\text {unranked }}=\frac{1}{\text { length(list })}\right)$. Then, combined scores across all enriched resource lists were computed. 
Finally, we drew 10 million random input lists and scored genes analogously to estimate the background distribution of scores, compute resampling based enrichment p-values and to adjust for multiple testing by the Benjamini Hochberg procedure (FDR $<0.05)$.

\section{Direct Mutation analysis of chondrodysplasia patients}

DNA was extracted by standard protocols from fetal fibroblasts and FFPE (Formalin-fixed paraffinembedded) tissue (Qiagen). PCR-amplifications of exons and intron-exon boundaries of TTC30A (ENST00000355689.6) and TTC30B (ENST00000408939.4) were analyzed with an ABI3130xI capillary sequencer (Life Technologies) to detect putative mutations ${ }^{140,141}$. Allele frequency was bioinformatically analyzed using an in-house exome database, the 1000 Genomes Project (http://www.internationalgenome.org/), the NHLBI Exome Sequencing Project (http://evs.gs.washington.edu/EVS/), and the dbSNP database (https://www.ncbi.nlm.nih.gov/projects/SNP/).

\section{Whole exome sequencing of chondrodysplasia patients}

The SureSelect Exome Enrichment kit V7 (Agilent Technologies) was used to enrich exome sequences. DNA was sequenced with a NextSeq 550 System (Illumina) resulting in 2x150-bp paired-end reads. A workflow based on the Genome Analysis Toolkit best practice was used to analyze the sequencing results bioinformatically ${ }^{142}$. For annotation and further processing the Ensembl Variant Effect Predictor software and GEMININ were used ${ }^{143-145}$.

\section{Whole exome sequencing of nephronophthisis patients}

Following informed consent, we obtained clinical data, pedigree data, and blood samples from individuals with nephronophthisis-related ciliopathies (NPHP-RC) from worldwide sources using a standardized questionnaire. Approval for human subject research was obtained from the Institutional Review Boards of the University of Michigan and Boston Children's Hospital. Informed consent was obtained from the individuals and/or legal guardians, as appropriate. The diagnosis of NPHP-RC was made by nephrologists based on relevant imaging.

Whole exome sequencing (WES) was performed as previously described ${ }^{146}$. Briefly, DNA samples from affected individuals and unaffected family members were subjected to WES using Agilent SureSelect ${ }^{\mathrm{TM}}$ human exome capture arrays (Life Technologies) with next generation sequencing (NGS) on an Illumina ${ }^{\mathrm{TM}}$ sequencing platform. Sequence reads were mapped against the human reference genome (NCBI build 37/hg19) using CLC Genomics Workbench (version 5.0.1) software (QIAGEN). Mutation analysis was performed under recessive, dominant or de novo models, as previously published ${ }^{146-148}$.

\section{Statistical analysis}

Volume, number of kidney cysts and total kidney volume (excluding cysts) between the injected side of the control (slc45a2) and the injected side of ift80, ift172 or ttc30a targeted animals, respectively, were compared with the Mann-Whitney-U test. The same test was used for analysis of eye distance, cilia area measurements, and pronephros length (Lectin) measurements. The paired t-test was used for 
statistical analysis of limb length. Unpaired t-test statistics were calculated for centrosome number after testing for normal distribution. Differences of edema formation and excretion were analyzed using chi test. Statistical analysis was performed with GraphPad Prism 8.3.1. $\left({ }^{* \star * *} p<0.0001,{ }^{* * *} p<0.001\right.$, ${ }^{* *} p<0.01$, and $\left.{ }^{*} p<0.05\right)$. All measurements were taken from distinct samples. No sample-size calculation was performed before the experiments.

\section{Data availability statement}

The datasets generated during and/or analysed during the current study are available from the corresponding author on reasonable request. The raw data associated with Figure 3a and Supp. Figures 3 c,d was deposited at at SRA with the BioProject accession number: PRJNA670560.

\section{Code availability statement}

The code used in the in silico screen is publicly available at https://github.com/genepi-freiburg/GeneSet.

\section{ACKNOWLEDGEMENTS}

We are grateful for technical assistance to Alena Sammarco, Jessika Kleindienst, Marko Vujanovic and Claudia Meyer, and the staff of the Life Imaging Center Freiburg at the Center for Biological Systems Analysis (ZBSA) of the University of Freiburg, the Center for Microscopy and Image Analysis (ZMB) and the Zurich Integrative Rodent Physiology (ZIRP) of the University of Zurich. This study was supported by the German Research Foundation (DFG) to SSL (Emmy Noether Program (Li-Li 1817/21), PS (CRC 992) and AK (KO 3598-5/1), and the Swiss National Science Foundation (SNF) to SSL (NCCR Kidney.CH) and (310030_189102/1). FH was supported by the National Institutes of Health (DK_068306).

\section{AUTHOR CONTRIBUTIONS}

S.S.L. and M.G. wrote the manuscript with contributions from A.H., P.S., K.G., K.D., F.Hi., E.L. and A.K.. S.S.L. supervised the project. M.G. performed most experiments and analyzed most of the data. S.S., R.D., F.He., and W.S. contributed to Xenopus experiments and data analysis. A.K., A.H., and P.S. performed and analyzed data for the in silico screen. K.G. analyzed the RNA-Seq and scRNA-Seq data. F.Hi., K.D. and E.L. screened patient cohorts for TTC30A/TTC30B mutations.

\section{COMPETING INTERESTS STATEMENT}

The authors declare no competing interests.

\section{REFERENCES}

1. Kempeneers, C. \& Chilvers, M. A. To beat, or not to beat, that is question! The spectrum of 
ciliopathies. Pediatr. Pulmonol. 53, 1122-1129 (2018).

2. Braun, D. A. \& Hildebrandt, F. Ciliopathies. Cold Spring Harb. Perspect. Biol. 9, a028191 (2017).

3. Chen, H. Y., Kelley, R. A., Li, T. \& Swaroop, A. Primary cilia biogenesis and associated retinal $\begin{array}{lllll}\text { ciliopathies. Semin. Cell Dev. Biol. S1084952119301673 (2020) } & \end{array}$ doi:10.1016/j.semcdb.2020.07.013.

4. Schmidts, M. \& Mitchison, H. M. Severe skeletal abnormalities caused by defects in retrograde intraflagellar transport dyneins. in Dyneins 356-401 (Elsevier, 2018). doi:10.1016/B978-0-12809470-9.00015-1.

5. Nachury, M. V. et al. A Core Complex of BBS Proteins Cooperates with the GTPase Rab8 to Promote Ciliary Membrane Biogenesis. Cell 129, 1201-1213 (2007).

6. Hoff, S. et al. ANKS6 is a central component of a nephronophthisis module linking NEK8 to INVS and NPHP3. Nat. Genet. 45, 951-956 (2013).

7. Handa, A., Voss, U., Hammarsjö, A., Grigelioniene, G. \& Nishimura, G. Skeletal ciliopathies: a pattern recognition approach. Jpn. J. Radiol. 38, 193-206 (2020).

8. Geister, K. A. \& Camper, S. A. Advances in Skeletal Dysplasia Genetics. Annu. Rev. Genomics Hum. Genet. 16, 199-227 (2015).

9. Schmidts, M. Clinical genetics and pathobiology of ciliary chondrodysplasias. J. Pediatr. Genet. 03, 049-064 (2015).

10. Aldahmesh, M. A. et al. IFT27, encoding a small GTPase component of IFT particles, is mutated in a consanguineous family with Bardet-Biedl syndrome. Hum. Mol. Genet. 23, 3307-3315 (2014).

11. Lindstrand, A. et al. Copy-Number Variation Contributes to the Mutational Load of Bardet-Biedl Syndrome. Am. J. Hum. Genet. 99, 318-336 (2016).

12. Mick, D. U. et al. Proteomics of Primary Cilia by Proximity Labeling. Dev. Cell 35, 497-512 (2015).

13. Kohli, P. et al. The ciliary membrane-associated proteome reveals actin-binding proteins as key components of cilia. EMBO Rep. 18, 1521-1535 (2017).

14. Ishikawa, H., Thompson, J., Yates, J. R. \& Marshall, W. F. Proteomic analysis of mammalian primary cilia. Curr. Biol. CB 22, 414-419 (2012).

15. Pathak, N., Austin-Tse, C. A., Liu, Y., Vasilyev, A. \& Drummond, I. A. Cytoplasmic carboxypeptidase 5 regulates tubulin glutamylation and zebrafish cilia formation and function. Mol. Biol. Cell 25, 1836-1844 (2014).

16. Gadadhar, S. et al. Tubulin glycylation controls primary cilia length. J. Cell Biol. 216, 2701-2713 (2017).

17. Roll-Mecak, A. The Tubulin Code in Microtubule Dynamics and Information Encoding. Dev. Cell 54, 7-20 (2020).

18. $\mathrm{He}, \mathrm{K}$. et al. Axoneme polyglutamylation regulated by Joubert syndrome protein ARL13B controls ciliary targeting of signaling molecules. Nat. Commun. 9, 3310 (2018).

19. Lee, J. E. et al. CEP41 is mutated in Joubert syndrome and is required for tubulin glutamylation at the cilium. Nat. Genet. 44, 193-199 (2012).

20. Huangfu, D. et al. Hedgehog signalling in the mouse requires intraflagellar transport proteins. Nature 426, 83-87 (2003).

21. Liu, A., Wang, B. \& Niswander, L. A. Mouse intraflagellar transport proteins regulate both the activator and repressor functions of Gli transcription factors. Dev. Camb. Engl. 132, 3103-3111 (2005).

22. Hoover, A. N. et al. $\mathrm{C} 2 \mathrm{~cd} 3$ is required for cilia formation and Hedgehog signaling in mouse. Development 135, 4049-4058 (2008).

23. Stottmann, R. W., Tran, P. V., Turbe-Doan, A. \& Beier, D. R. Ttc21b is required to restrict sonic hedgehog activity in the developing mouse forebrain. Dev. Biol. 335, 166-178 (2009).

24. Rix, S., Calmont, A., Scambler, P. J. \& Beales, P. L. An Ift80 mouse model of short rib polydactyly syndromes shows defects in hedgehog signalling without loss or malformation of cilia. Hum. Mol. Genet. 20, 1306-1314 (2011).

25. McInerney-Leo, A. M. et al. Short-Rib Polydactyly and Jeune Syndromes Are Caused by Mutations in WDR60. Am. J. Hum. Genet. 93, 515-523 (2013).

26. Paige Taylor, S. et al. An inactivating mutation in intestinal cell kinase, ICK, impairs hedgehog signalling and causes short rib-polydactyly syndrome. Hum. Mol. Genet. 25, 3998-4011 (2016).

27. University of Washington Center for Mendelian Genomics Consortium et al. Mutations in DYNC2LI1 disrupt cilia function and cause short rib polydactyly syndrome. Nat. Commun. 6, 7092 (2015).

28. Wu, C. et al. Talpid3-Binding Centrosomal Protein Cep120 Is Required for Centriole Duplication and Proliferation of Cerebellar Granule Neuron Progenitors. PLOS ONE 9, e107943 (2014).

29. Caparrós-Martín, J. A. et al. The ciliary Evc/Evc2 complex interacts with Smo and controls 
Hedgehog pathway activity in chondrocytes by regulating Sufu/Gli3 dissociation and Gli3 trafficking in primary cilia. Hum. Mol. Genet. 22, 124-139 (2013).

30. Alby, C. et al. Mutations in KIAA0586 Cause Lethal Ciliopathies Ranging from a Hydrolethalus Phenotype to Short-Rib Polydactyly Syndrome. Am. J. Hum. Genet. 97, 311-318 (2015).

31. Wu, C., Li, J., Peterson, A., Tao, K. \& Wang, B. Loss of dynein-2 intermediate chain Wdr34 results in defects in retrograde ciliary protein trafficking and Hedgehog signaling in the mouse. Hum. Mol. Genet. 26, 2386-2397 (2017).

32. Frikstad, K.-A. M. et al. A CEP104-CSPP1 Complex Is Required for Formation of Primary Cilia Competent in Hedgehog Signaling. Cell Rep. 28, 1907-1922.e6 (2019).

33. Anvarian, Z., Mykytyn, K., Mukhopadhyay, S., Pedersen, L. B. \& Christensen, S. T. Cellular signalling by primary cilia in development, organ function and disease. Nat. Rev. Nephrol. 15, 199-219 (2019).

34. Bangs, F. \& Anderson, K. V. Primary Cilia and Mammalian Hedgehog Signaling. Cold Spring Harb. Perspect. Biol. 9, a028175 (2017).

35. St-Jacques, B., Hammerschmidt, M. \& McMahon, A. P. Indian hedgehog signaling regulates proliferation and differentiation of chondrocytes and is essential for bone formation. Genes Dev. 13, 2072-2086 (1999).

36. Litingtung, Y., Dahn, R. D., Li, Y., Fallon, J. F. \& Chiang, C. Shh and Gli3 are dispensable for limb skeleton formation but regulate digit number and identity. Nature 418, 979-983 (2002).

37. Cavalcanti, D. P. et al. Mutation in IFT80 in a fetus with the phenotype of Verma-Naumoff provides molecular evidence for Jeune-Verma-Naumoff dysplasia spectrum. J. Med. Genet. 48, 88-92 (2011).

38. Bizaoui, V. et al. Mutations in IFT80 cause SRPS Type IV. Report of two families and review. Am. J. Med. Genet. A. 179, 639-644 (2019).

39. Beales, P. L. et al. IFT80, which encodes a conserved intraflagellar transport protein, is mutated in Jeune asphyxiating thoracic dystrophy. Nat. Genet. 39, 727-729 (2007).

40. Halbritter, J. et al. Defects in the IFT-B Component IFT172 Cause Jeune and Mainzer-Saldino Syndromes in Humans. Am. J. Hum. Genet. 93, 915-925 (2013).

41. Schaefer, E. et al. Identification of a novel mutation confirms the implication of IFT172 (BBS20) in Bardet-Biedl syndrome. J. Hum. Genet. 61, 447-450 (2016).

42. Bujakowska, K. M. et al. Mutations in IFT172 cause isolated retinal degeneration and BardetBiedl syndrome. Hum. Mol. Genet. 24, 230-242 (2015).

43. Taschner, M. et al. Intraflagellar transport proteins $172,80,57,54,38$, and 20 form a stable tubulin-binding IFT -B2 complex. EMBO J. 35, 773-790 (2016).

44. Katoh, Y. et al. Overall Architecture of the Intraflagellar Transport (IFT)-B Complex Containing Cluap1/IFT38 as an Essential Component of the IFT-B Peripheral Subcomplex. J. Biol. Chem. 291, 10962-10975 (2016).

45. Hudak, L. M. et al. The Intraflagellar Transport Protein Ift80 Is Essential for Photoreceptor Survival in a Zebrafish Model of Jeune Asphyxiating Thoracic Dystrophy. Invest. Ophthalmol. Vis. Sci. 51, 3792-3799 (2010).

46. Gorivodsky, M. et al. Intraflagellar transport protein 172 is essential for primary cilia formation and plays a vital role in patterning the mammalian brain. Dev. Biol. 325, 24-32 (2009).

47. Friedland-Little, J. M. et al. A novel murine allele of Intraflagellar Transport Protein 172 causes a syndrome including VACTERL-like features with hydrocephalus. Hum. Mol. Genet. 20, 37253737 (2011).

48. Yuan, X. et al. Ciliary IFT80 balances canonical versus non-canonical hedgehog signalling for osteoblast differentiation. Nat. Commun. 7, 11024 (2016).

49. Howard, P. W., Howard, T. L. \& Maurer, R. A. Generation of mice with a conditional allele for Ift172. Transgenic Res. 19, 121-126 (2010).

50. Li, X., Yang, S., Han, L., Mao, K. \& Yang, S. Ciliary IFT80 is essential for intervertebral disc development and maintenance. FASEB J. 34, 6741-6756 (2020).

51. Shimizu-Nishikawa, K., Takahashi, J. \& Nishikawa, A. Intercalary and supernumerary regeneration in the limbs of the frog,Xenopus laevis. Dev. Dyn. 227, 563-572 (2003).

52. Christen, B., Rodrigues, A. M. C., Monasterio, M. B., Roig, C. F. \& Belmonte, J. C. I. Transient downregulation of Bmp signalling induces extra limbs in vertebrates. Development 139, 25572565 (2012).

53. Wang, F. et al. Targeted gene disruption in Xenopus laevis using CRISPR/Cas9. Cell Biosci. 5, 15 (2015).

54. Stopper, G. F., Richards-Hrdlicka, K. L. \& Wagner, G. P. Hedgehog inhibition causes complete loss of limb outgrowth and transformation of digit identity in Xenopus tropicalis: HEDGEHOG 
FUNCTION IN XENOPUS LIMB DEVELOPMENT. J. Exp. Zoolog. B Mol. Dev. Evol. 326, 110124 (2016).

55. Wu, R. S. et al. A Rapid Method for Directed Gene Knockout for Screening in G0 Zebrafish. Dev. Cell 46, 112-125.e4 (2018).

56. Hoshijima, K. et al. Highly Efficient CRISPR-Cas9-Based Methods for Generating Deletion Mutations and F0 Embryos that Lack Gene Function in Zebrafish. Dev. Cell 51, 645-657.e4 (2019).

57. Jao, L.-E., Wente, S. R. \& Chen, W. Efficient multiplex biallelic zebrafish genome editing using a CRISPR nuclease system. Proc. Natl. Acad. Sci. 110, 13904-13909 (2013).

58. Banach, M., Edholm, E.-S. \& Robert, J. Exploring the functions of nonclassical MHC class Ib genes in Xenopus laevis by the CRISPR/Cas9 system. Dev. Biol. 426, 261-269 (2017).

59. DeLay, B. D. et al. Tissue-Specific Gene Inactivation in Xenopus laevis: Knockout of Ihx1 in the Kidney with CRISPR/Cas9. Genetics 208, 673-686 (2018).

60. Blitz, I. L., Biesinger, J., Xie, X. \& Cho, K. W. Y. Biallelic genome modification in F o Xenopus tropicalis embryos using the CRISPR/Cas system: Biallelic Genome Modification. genesis 51, 827-834 (2013).

61. Naert, T. et al. CRISPR/Cas9 mediated knockout of rb1 and rbl1 leads to rapid and penetrant retinoblastoma development in Xenopus tropicalis. Sci. Rep. 6, 35264 (2016).

62. Szenker-Ravi, E. et al. RSPO2 inhibition of RNF43 and ZNRF3 governs limb development independently of LGR4/5/6. Nature 557, 564-569 (2018).

63. Kariminejad, A. et al. Homozygous Null TBX4 Mutations Lead to Posterior Amelia with Pelvic and Pulmonary Hypoplasia. Am. J. Hum. Genet. 105, 1294-1301 (2019).

64. Shigeta, M. et al. Rapid and efficient analysis of gene function using CRISPR-Cas9 in Xenopus tropicalis founders. Genes Cells 21, 755-771 (2016).

65. Hayashi, S. et al. Evidence for an amphibian sixth digit. Zool. Lett. 1, 17 (2015).

66. Casteels, I., Demandt, E. \& Legius, E. Visual loss as the presenting sign of Jeune syndrome. Eur. J. Paediatr. Neurol. 4, 243-247 (2000).

67. Yuan, X. \& Yang, S. Primary Cilia and Intraflagellar Transport Proteins in Bone and Cartilage. J. Dent. Res. 95, 1341-1349 (2016).

68. Yuan, X. \& Yang, S. Deletion of IFT80 Impairs Epiphyseal and Articular Cartilage Formation Due to Disruption of Chondrocyte Differentiation. PLOS ONE 10, e0130618 (2015).

69. Duran, I. et al. Destabilization of the IFT-B cilia core complex due to mutations in IFT81 causes a Spectrum of Short-Rib Polydactyly Syndrome. Sci. Rep. 6, 34232 (2016).

70. Duran, I. et al. Mutations in IFT-A satellite core component genes IFT43 and IFT121 produce short rib polydactyly syndrome with distinctive campomelia. Cilia 6, 7 (2017).

71. Lunt, S. C., Haynes, T. \& Perkins, B. D. Zebrafish ift57, ift88, and ift172 intraflagellar transport mutants disrupt cilia but do not affect hedgehog signaling. Dev. Dyn. 238, 1744-1759 (2009).

72. Sims-Mourtada, J., Izzo, J. G., Ajani, J. \& Chao, K. S. C. Sonic Hedgehog promotes multiple drug resistance by regulation of drug transport. Oncogene 26, 5674-5679 (2007).

73. Corrêa, S. et al. $A B C B 1$ regulation through LRPPRC is influenced by the methylation status of the GC -100 box in its promoter. Epigenetics 9, 1172-1183 (2014).

74. Chen, Y., Bieber, M. M. \& Teng, N. N. H. Hedgehog signaling regulates drug sensitivity by targeting $A B C$ transporters $A B C B 1$ and $A B C G 2$ in epithelial ovarian cancer. Mol. Carcinog. n/an/a (2013) doi:10.1002/mc.22015.

75. Sun, J. et al. The Effects of Indian Hedgehog Deletion on Mesenchyme Cells: Inducing Intermediate Cartilage Scaffold Ossification to Cause Growth Plate and Phalange Joint Absence, Short Limb, and Dwarfish Phenotypes. Stem Cells Dev. 27, 1412-1425 (2018).

76. $\mathrm{Li}, \mathrm{H}$. et al. Olfactomedin 4 deficiency promotes prostate neoplastic progression and is associated with upregulation of the hedgehog-signaling pathway. Sci. Rep. 5, 16974 (2015).

77. Tariki, M. et al. RIO kinase 3 acts as a SUFU-dependent positive regulator of Hedgehog signaling. Cell. Signal. 25, 2668-2675 (2013).

78. Xiao, Q. et al. AMP-activated protein kinase-dependent autophagy mediated the protective effect of sonic hedgehog pathway on oxygen glucose deprivation-induced injury of cardiomyocytes. Biochem. Biophys. Res. Commun. 457, 419-425 (2015).

79. Matsumoto, N. et al. C3a Enhances the Formation of Intestinal Organoids through C3aR1. Front. Immunol. 8, 1046 (2017).

80. Zhang, Y., Laterra, J. \& Pomper, M. G. Hedgehog Pathway Inhibitor HhAntag691 Is a Potent Inhibitor of ABCG2/BCRP and ABCB1/Pgp. Neoplasia 11, 96-101 (2009).

81. Liu, W. et al. Olfactomedin 4 deletion induces colon adenocarcinoma in ApcMin/+ mice. Oncogene 35, 5237-5247 (2016). 
82. Jati, S. et al. Wnt5A Signaling Promotes Defense Against Bacterial Pathogens by Activating a Host Autophagy Circuit. Front. Immunol. 9, 679 (2018).

83. Zhang, W. et al. Expanding the genetic architecture and phenotypic spectrum in the skeletal ciliopathies. Hum. Mutat. 39, 152-166 (2018).

84. Silveira, K. C., Moreno, C. A. \& Cavalcanti, D. P. Beemer-Langer syndrome is a ciliopathy due to biallelic mutations in IFT122. Am. J. Med. Genet. A. 173, 1186-1189 (2017).

85. Mendes Maia, T., Gogendeau, D., Pennetier, C., Janke, C. \& Basto, R. Bug22 influences cilium morphology and the post-translational modification of ciliary microtubules. Biol. Open 3, 138-151 (2014).

86. Meng, D., Cao, M., Oda, T. \& Pan, J. The conserved ciliary protein Bug22 controls planar beating of Chlamydomonas flagella. J. Cell Sci. 127, 281-287 (2014).

87. Takahara, M. et al. C11ORF74 interacts with the IFT-A complex and participates in ciliary BBSome localization. J. Biochem. (Tokyo) 165, 257-267 (2019).

88. Joukov, V., De Nicolo, A., Rodriguez, A., Walter, J. C. \& Livingston, D. M. Centrosomal protein of 192 kDa (Cep192) promotes centrosome-driven spindle assembly by engaging in organellespecific Aurora A activation. Proc. Natl. Acad. Sci. 107, 21022-21027 (2010).

89. Gomez-Ferreria, M. A., Bashkurov, M., Mullin, M., Gingras, A.-C. \& Pelletier, L. CEP192 interacts physically and functionally with the K63-deubiquitinase CYLD to promote mitotic spindle assembly. Cell Cycle 11, 3555-3558 (2012).

90. Fan, Z.-C. et al. Chlamydomonas IFT70/CrDYF-1 Is a Core Component of IFT Particle Complex B and Is Required for Flagellar Assembly. Mol. Biol. Cell 21, 2696-2706 (2010).

91. Pathak, N., Obara, T., Mangos, S., Liu, Y. \& Drummond, I. A. The Zebrafish fleer Gene Encodes an Essential Regulator of Cilia Tubulin Polyglutamylation. Mol. Biol. Cell 18, 4353-4364 (2007).

92. Pathak, N., Austin, C. A. \& Drummond, I. A. Tubulin Tyrosine Ligase-like Genes $t t / 13$ and $t t / 16$ Maintain Zebrafish Cilia Structure and Motility. J. Biol. Chem. 286, 11685-11695 (2011).

93. Kelly, N. H., Huynh, N. P. T. \& Guilak, F. Single cell RNA-sequencing reveals cellular heterogeneity and trajectories of lineage specification during murine embryonic limb development. Matrix Biol. 89, 1-10 (2020).

94. Howe, K. et al. The zebrafish reference genome sequence and its relationship to the human genome. Nature 496, 498-503 (2013).

95. Session, A. M. et al. Genome evolution in the allotetraploid frog Xenopus laevis. Nature 538, 336343 (2016).

96. Yang, S. Cilia Ift protein and motor -related bone diseases and mouse models. Front. Biosci. 20, 515-555 (2015).

97. Yang, J., Andre, P., Ye, L. \& Yang, Y.-Z. The Hedgehog signalling pathway in bone formation. Int. J. Oral Sci. 7, 73-79 (2015).

98. Gao, B. et al. Mutations in $\mathrm{IHH}$, encoding Indian hedgehog, cause brachydactyly type A-1. Nat. Genet. 28, 386-388 (2001).

99. Hwang, S. et al. The G protein-coupled receptor Gpr161 regulates forelimb formation, limb patterning and skeletal morphogenesis in a primary cilium-dependent manner. Development 145, dev154054 (2018).

100. Geetha-Loganathan, P., Nimmagadda, S. \& Scaal, M. Wnt signaling in limb organogenesis. Organogenesis 4, 109-115 (2008).

101. Kuss, P. et al. Regulation of cell polarity in the cartilage growth plate and perichondrium of metacarpal elements by HOXD13 and WNT5A. Dev. Biol. 385, 83-93 (2014).

102. Saari, J., Lovell, M. A., Yu, H.-C. \& Bellus, G. A. Compound heterozygosity for a frame shift mutation and a likely pathogenic sequence variant in the planar cell polarity-ciliogenesis gene WDPCP in a girl with polysyndactyly, coarctation of the aorta, and tongue hamartomas. Am. J. Med. Genet. A. 167, 421-427 (2015).

103. Capellini, T. D. Pbx1/Pbx2 requirement for distal limb patterning is mediated by the hierarchical control of Hox gene spatial distribution and Shh expression. Development 133, 2263-2273 (2006).

104. Brison, N., Debeer, P. \& Tylzanowski, P. Joining the fingers: A HOXD13 story: Synpolydactyly and HOXD13 Gene Mutations. Dev. Dyn. 243, 37-48 (2014).

105. Sim, H. J. et al. Simple Method To Characterize the Ciliary Proteome of Multiciliated Cells. J. Proteome Res. 19, 391-400 (2020).

106. SYSCILIA Study Group et al. The SYSCILIA gold standard (SCGSv1) of known ciliary components and its applications within a systems biology consortium. Cilia 2, 7 (2013).

107. El-Brolosy, M. A. et al. Genetic compensation triggered by mutant mRNA degradation. Nature 568, 193-197 (2019). 
108. Sztal, T. E. \& Stainier, D. Y. R. Transcriptional adaptation: a mechanism underlying genetic robustness. Dev. Camb. Engl. 147, (2020).

109. Drummond, I. A. et al. Early development of the zebrafish pronephros and analysis of mutations affecting pronephric function. Dev. Camb. Engl. 125, 4655-4667 (1998).

110. Taschner, M., Kotsis, F., Braeuer, P., Kuehn, E. W. \& Lorentzen, E. Crystal structures of IFT70/52 and IFT52/46 provide insight into intraflagellar transport B core complex assembly. J. Cell Biol. 207, 269-282 (2014).

111. Takei, R., Katoh, Y. \& Nakayama, K. Robust interaction of IFT70 with IFT52-IFT88 in the IFT-B complex is required for ciliogenesis. Biol. Open 7, bio033241 (2018).

112. Ou, G., E. Blacque, O., Snow, J. J., Leroux, M. R. \& Scholey, J. M. Functional coordination of intraflagellar transport motors. Nature 436, 583-587 (2005).

113. Hong, S.-R. et al. Spatiotemporal manipulation of ciliary glutamylation reveals its roles in intraciliary trafficking and Hedgehog signaling. Nat. Commun. 9, 1732 (2018).

114. O'Hagan, R. et al. Glutamylation Regulates Transport, Specializes Function, and Sculpts the Structure of Cilia. Curr. Biol. CB 27, 3430-3441.e6 (2017).

115. Uhlén, M. et al. Proteomics. Tissue-based map of the human proteome. Science 347, 1260419 (2015).

116. Cao, J. et al. The single-cell transcriptional landscape of mammalian organogenesis. Nature 566, 496-502 (2019).

117. Du, Y. et al. A rare TTC30B variant is identified as a candidate for synpolydactyly in a Chinese pedigree. Bone 127, 503-509 (2019).

118. Ma, M., Legué, E., Tian, X., Somlo, S. \& Liem, K. F. Cell-Autonomous Hedgehog Signaling Is Not Required for Cyst Formation in Autosomal Dominant Polycystic Kidney Disease. J. Am. Soc. Nephrol. JASN 30, 2103-2111 (2019).

119. Normal table of Xenopus laevis (Daudin): a systematical and chronological survey of the development from the fertilized egg till the end of metamorphosis. (Garland Pub, 1994).

120. Kaminski, M. M. et al. Direct reprogramming of fibroblasts into renal tubular epithelial cells by defined transcription factors. Nat. Cell Biol. 18, 1269-1280 (2016).

121. Ulmer, B. et al. Calponin 2 Acts As an Effector of Noncanonical Wnt-Mediated Cell Polarization during Neural Crest Cell Migration. Cell Rep. 3, 615-621 (2013).

122. Walentek, P., Beyer, T., Thumberger, T., Schweickert, A. \& Blum, M. ATP4a Is Required for WntDependent Foxj1 Expression and Leftward Flow in Xenopus Left-Right Development. Cell Rep. 1, 516-527 (2012).

123. Park, T. J., Haigo, S. L. \& Wallingford, J. B. Ciliogenesis defects in embryos lacking inturned or fuzzy function are associated with failure of planar cell polarity and Hedgehog signaling. Nat. Genet. 38, 303-311 (2006).

124. Cerqueira, D. M., Tran, U., Romaker, D., Abreu, J. G. \& Wessely, O. Sterol carrier protein 2 regulates proximal tubule size in the Xenopus pronephric kidney by modulating lipid rafts. Dev. Biol. 394, 54-64 (2014).

125. Labun, K. et al. CHOPCHOP v3: expanding the CRISPR web toolbox beyond genome editing. Nucleic Acids Res. 47, W171-W174 (2019).

126. Doench, J. G. et al. Rational design of highly active sgRNAs for CRISPR-Cas9-mediated gene inactivation. Nat. Biotechnol. 32, 1262-1267 (2014).

127. Nakayama, T. et al. Simple and efficient CRISPR/Cas9-mediated targeted mutagenesis in Xenopus tropicalis: CRISPR-Cas Genome Editing in Xenopus. genesis 51, 835-843 (2013).

128. Park, T. J., Mitchell, B. J., Abitua, P. B., Kintner, C. \& Wallingford, J. B. Dishevelled controls apical docking and planar polarization of basal bodies in ciliated epithelial cells. Nat. Genet. 40, 871879 (2008).

129. Naert, T. \& Vleminckx, K. CRISPR/Cas9 disease models in zebrafish and Xenopus: The genetic renaissance of fish and frogs. Drug Discov. Today Technol. 28, 41-52 (2018).

130. Afgan, E. et al. The Galaxy platform for accessible, reproducible and collaborative biomedical analyses: 2016 update. Nucleic Acids Res. 44, W3-W10 (2016).

131. Dobin, A. et al. STAR: ultrafast universal RNA-seq aligner. Bioinforma. Oxf. Engl. 29, 15-21 (2013).

132. Liao, Y., Smyth, G. K. \& Shi, W. featureCounts: an efficient general purpose program for assigning sequence reads to genomic features. Bioinformatics 30, 923-930 (2014).

133. Stuart, T. et al. Comprehensive Integration of Single-Cell Data. Cell 177, 1888-1902.e21 (2019).

134. R Core Team (2020). R: A language and environment for statistical computing. R Foundation for Statistical Computing, Vienna, Austria.

135. Sive, H. L., Grainger, R. \& Harland, R. M. Early development of Xenopus laevis: a laboratory 
manual. (Cold Spring Harbor Laboratory Press., 2010).

136. Lienkamp, S. S. et al. Vertebrate kidney tubules elongate using a planar cell polarity-dependent, rosette-based mechanism of convergent extension. Nat. Genet. 44, 1382-1387 (2012).

137. Lienkamp, S. et al. Inversin relays Frizzled-8 signals to promote proximal pronephros development. Proc. Natl. Acad. Sci. 107, 20388-20393 (2010).

138. Hopkins, T. M. et al. Combining micro-computed tomography with histology to analyze biomedical implants for peripheral nerve repair. J. Neurosci. Methods 255, 122-130 (2015).

139. Benjamini, Y. \& Hochberg, Y. Controlling the False Discovery Rate: A Practical and Powerful Approach to Multiple Testing. J. R. Stat. Soc. Ser. B Methodol. 57, 289-300 (1995).

140. Hoffmann, K. et al. Mutations in the gene encoding the lamin B receptor produce an altered nuclear morphology in granulocytes (Pelger-Huët anomaly). Nat. Genet. 31, 410-414 (2002).

141. Smits, P. et al. Lethal Skeletal Dysplasia in Mice and Humans Lacking the Golgin GMAP-210. N. Engl. J. Med. 362, 206-216 (2010).

142. DePristo, M. A. et al. A framework for variation discovery and genotyping using next-generation DNA sequencing data. Nat. Genet. 43, 491-498 (2011).

143. McLaren, W. et al. The Ensembl Variant Effect Predictor. Genome Biol. 17, 122 (2016).

144. Paila, U., Chapman, B. A., Kirchner, R. \& Quinlan, A. R. GEMINI: Integrative Exploration of Genetic Variation and Genome Annotations. PLoS Comput. Biol. 9, e1003153 (2013).

145. Wehrle, A. et al. A common pathomechanism in GMAP-210- and LBR-related diseases. $\mathrm{JCl}$ Insight 3, e121150 (2018).

146. van der Ven, A. T. et al. Whole-Exome Sequencing Identifies Causative Mutations in Families with Congenital Anomalies of the Kidney and Urinary Tract. J. Am. Soc. Nephrol. 29, 2348-2361 (2018).

147. Vivante, A. et al. Exome Sequencing Discerns Syndromes in Patients from Consanguineous Families with Congenital Anomalies of the Kidneys and Urinary Tract. J. Am. Soc. Nephrol. 28, 69-75 (2017).

148. Warejko, J. K. et al. Whole Exome Sequencing of Patients with Steroid-Resistant Nephrotic Syndrome. Clin. J. Am. Soc. Nephrol. 13, 53-62 (2018).

\section{Figure legends}

Figure 1: CRISPR targeting of ift 80 and ift172 leads to skeletal defects in $X$. tropicalis

(a, b) Analysis of edema formation in ift80 and ift172 mutants at stage 41/42. Note the smaller eyes in ift80 targeted embryos. (c - e) Limb phenotypes in post-metamorphic froglets. MicroCT scans of representative mutant froglets of slc45a2, ift80 and ift172 are shown in dorsal views. Injections of RNPs were performed unilaterally (left) at the 2-cell stage. For the same animals 3D-reconstructions are pictured. Limb muscles are shown in red, eyes in yellow.

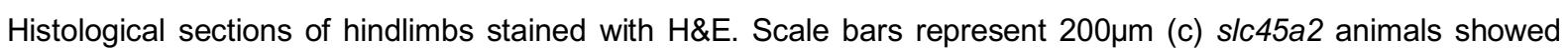
regular limb development. (d) Polydactyly is indicated by orange arrowheads to mark black nailed digits. MicroCT scans and histological stainings revealed cartilage accumulations in CRISPR-targeted froglets (green arrowheads). 3D-reconstructions demonstrate that only left limbs were affected (red arrowheads). Length of respective uninjected (green dots) vs injected (red dots) limbs of the same individual are plotted (grey lines). $\mathrm{R}$ - resting zone; $\mathrm{P}$ - proliferating zone; $\mathrm{PH}$ - pre hypertrophic zone; $\mathrm{H}$ - hypertrophic zone. $\mathrm{p}>0.05 \mathrm{~ns}$ (not significant); $\mathrm{p}<0.05$ *; $p<0.01^{* *} ; p<0.001^{* * *}$

Figure 2: Cystic kidney disease and cilia defects in ift80- and ift172 CRISPR targeted $X$. tropicalis.

(a - c) CRISPR/Cas9 targeting of s/c45a2, ift80 and ift172 were performed unilaterally in two-cell-stage embryos. Mesonephri of st. 61-63 froglets were analyzed by microCT scans and kidneys and cysts (red arrowheads in b, c) were segmented for 3D volumetric analysis ( $\mathrm{a}-\mathrm{c}$; red - injected; green - uninjected). (d) The number of cysts (> $0.2 \mathrm{~mm}$ ), (e) the ratio of total cyst volume (of cysts $>0.2 \mathrm{~mm}$ ) to kidney volume and (f) the total kidney volume (excluding cysts $>0.2 \mathrm{~mm}$ ) was calculated and compared between right untreated (-) and left injected (+) kidneys. (g) Whole mount in situ hybridization detects ift80 and ift172 in the multiciliated nephrostomes of the pronephros in 
$X$. laevis. (h) Schematic depiction of the embryonic renal system of Xenopus. (j, k) Excretion assay with fluoresceindextran at stage 38-40. Blue arrowheads point to the proximal part of the pronephros. Yellow arrowheads indicate fluorescence signal in the distal tubule, lacking on the injected side (j). $a-p$ : anterior - posterior.

(I) Confocal images of multiciliated epidermal cells (MCCs) stained against acetylated tubulin (cyan). Centrin-RFP fusion protein served as a lineage marker (red arrowheads) and indicates CRISPR-targeted MCCs. Blue arrowheads point to non-targeted (wild-type) cells. $(m)$ The ciliated area was determined for each cell.

Error bars indicate SEM. $p>0.05$ ns (not significant); $p<0.05^{*} ; p<0.01^{* *} ; p<0.001^{* * *}$

Figure 3: Transcriptomic profiling of ift80 and ift172 CRISPR targeted embryos and in silico screening for candidates with similar properties to identified SC-genes.

(a) Scatterplot of the log2-fold changes in mRNA expression between ift80- and ift172 targeted embryos. Significantly changed transcripts are labeled with gene names. Red dots mark genes exclusively altered in ift80or ift172 targeted embryos. Green dots indicate genes significantly changed in both conditions. Blue arrowheads point to genes for which an association with the hedgehog signaling pathway has been described, orange arrowheads on genes with wnt-associations. A table of respective associations and references thereof. (b) Schematic of the in silico screen. Known SC-disease genes serve as an input list. Resource lists represent datasets from various published screening approaches and are tested for enrichment of genes contained in the input list genes. Genes in significantly enriched resource lists are then scored based on membership or rank. The gene scores are statistically validated by an empirical $p$-value based on 10 million random drawings of input list. (c - $\mathrm{f}$ ) Whole mount in situ hybridizations for four candidate genes (c11orf74, cep192, cfap20, ttc30a) that were experimentally followed up are shown for tadpoles (nephrostome expression enlarged) and limb buds. (g) Resource Lists in which the candidate genes occurred.

Figure 4: $t t c 30$ loss of function resembles the SC-phenotype

(a) Phylogenetic tree of protein sequences of TTC30A/B orthologs in various species. Genetic duplication events are marked by red squares. (b, c) CRISPR targeting of ttc30a at the one-cell stage led to edema formation, quantified in (c). Co-injection of ttc30a mRNA partially rescued the phenotype.

(d) Unilaterally CRISPR targeted ttc30a froglets developed shortened limbs on the injected side (green arrowhead). MicroCT analysis and histological sections stained with H\&E demonstrated accumulation of cartilage (green

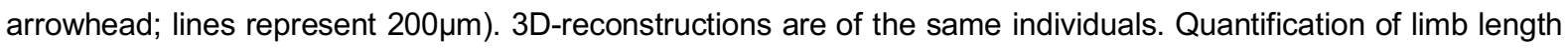
reduction of ttc30a targeted compared to non-targeted side. (e) MicroCT scans show cystic kidneys in ttc30a targeted animals (red arrowhead). 3D-reconstruction of the kidneys and cysts (red) were used for volumetric analysis. Quantifications of cyst number $(>0.2 \mathrm{~mm}$ ), the ratio of total cyst volume (of cysts $>0.2 \mathrm{~mm}$ ) to kidney volume and total kidney volume (excluding cysts $>0.2 \mathrm{~mm}$ ).

Figure 5: scRNA-Seq data analysis of $t t c 30 a / b$-positive cells in limbs

(a, b) Excretion assay of s/c45a2 sgRNA injected controls and ttc30a targeted embryos. Blue arrowheads point to the proximal tubule, yellow arrowheads highlight fluorescent dextran in the distal tubules as a measure of excretion.

(c) Confocal images of epidermal multiciliated cells. centrin-RFP (red) marks cells targeted for ttc30a (red arrowheads). Blue arrowheads label wild-type cells. Cilia are stained with acetylated gamma-tubulin (cyan). (d) Quantification of ciliated area per cell in slc45a2 and ttc30a CRISPR targeted cells.

Error bars indicate SEM. $p>0.05$ ns (not significant); $p<0.05 * ; p<0.01^{* *} ; p<0.001^{* * *}$

(e) Clustering of scRNA-Seq data from mice limbs of E15.5 into 10 different cell clusters using typical marker genes.

(f) Detection of Ttc30b expression in respective cell clusters. (g) Comparison of percentage of cells per cluster 
expressing Ttc30a1, Ttc30a2, Ttc30b, Ift80 and Ift172. (H) Expression level of Ttc30b, Ift80 and Ift172 in respective tissue clusters.

\section{Supplementary Figures}

Supplementary Figure 1: Specificity controls of ift80 and ift172 CRISPR/Cas 9 targeting experiments

(a) Gene structure of $X$. tropicalis ift80 and ift172 with introns (lines) and exons (boxes). sgRNA-binding-sites are marked by a red arrow. (b, c) CRISPR editing analysis for ift80 and ift172. The site of the expected cut is depicted by black vertical lines in Sanger Sequencing chromatograms, the sgRNA binding site is marked in blue and the PAM site in red. (d) Craniofacial defects of ift80 and ift172 CRISPR targeted embryos were analyzed by measuring the distance between the eyes of stage 43-46 $X$. tropicalis tadpoles and normalized to the average of uninjected controls. (e) Normalized eye distances decreased significantly in CRISPR targeted embryos. (f) Knockdown of ift80 and ift172 using anitsense morpholino oligonucleotides. Knockdown resulted in edema formation. (g, h) Decrease of eye-distance for ift80 and ift172 CRISPR targeted $X$. laevis embryos. (j) CRISPR targeting of ift80 and ift172 in $X$. laevis leads to edema formation, (k) In situ hybridization detected expression of ift80 and ift172 in limb buds of $X$. laevis (black arrowhead). po - posterior; a - anterior; pr - proximal; $d$ - distal (I) Categorization of mutant froglets according to phenotypic strength of limb malformations.

Error bars indicate SEM. $p>0.05$ ns (not significant); $p<0.05^{*} ; p<0.01^{* *} ; p<0.001^{* * *}$

Supplementary Figure 2: Phenotypic analysis of ift80 and ift172 targeted embryos.

(a, b) Expression analysis of ift80 and ift172 by whole mount in situ hybridization in st. 26/27 embryos. The magnified images show a spotty pattern on the epidermis. (c, d) Expression analysis of aplnr, nkx2.5, prox1, nkcc2 and sglt 1 of $X$. tropicalis (c) and $X$. laevis (d) unilaterally CRISPR targeted tadpoles. The bar graphs indicate a stronger (gray) or weaker (black) signal on the injected side.

$(e, f)$ Tomato-Lectin stain visualizes the pronephric tubules in unilaterally CRISPR targeted stage 39 tadpoles. Measurements of the bounding box area of the proximal tubule was not different between the injected side of the embryo and the wildtype half in each case. (g) Quantification of centrioles of epidermal MCCs after injection of centrin-RFP mRNA together with sgRNAs and Cas9. There was no significant difference in centriole number in CRISPR targeted cells. Error bars indicate SEM. $p>0.05$ ns (not significant); $p<0.05^{*} ; p<0.01^{* *} ; p<0.001^{* * *}$

Supplementary Figure 3: RNA-Seq analysis of ift80 and ift172 targeted embryos.

(a) Schematic representation of the RNA and DNA extraction protocol used for RN-Seq experiments. Embryos were genotyped before sending purified RNA for sequencing (see "materials and methods" for detailed description). (b) Indel- and knockout scores from ICE for experimental replicates used in the RNA-Seq experiment. (c, d) Vulcano-plots of RNA-Seq analysis of ift80 and ift172 targeted embryos show that the targeted genes were downregulated efficiently in the respective experiments (red arrowheads). (e) In situ hybridization for differentially expressed genes in the RNA-Seq screen. Col1a1 expression is shown for $X$. laevis, all others for $X$. tropicalis tadpoles. (f, g) In situ hybridization of tissue marker genes was examined in $X$. tropicalis (f) and $X$. laevis (g) after unilateral CRISPR targeting of ift80 and ift172. The quantifications indicate a stronger (gray) or weaker (black) signal on the injected side. No obvious changes were found, only the snail expression, marking cranial neural crest streams, appeared to be fused in ift80 and ift172 targeted embryos. 
bioRxiv preprint doi: https://doi.org/10.1101/2020.11.27.400994; this version posted November 27, 2020. The copyright holder for this preprint (which was not certified by peer review) is the author/funder, who has granted bioRxiv a license to display the preprint in perpetuity. It is made available under aCC-BY-NC-ND 4.0 International license.

Supplementary Figure 4: Analysis of SC-associated candidate genes

(a) Gene structure of $X$. tropicalis ttc30a with introns as lines and exons as boxes. The sgRNA target site is marked by a red arrow. (b) Genotyping and ICE analysis of ttc30a targeted embryos (sgRNA binding site - blue; PAM - red; cutting site - vertical black line). (c, d) Percentage of embryos developing edema after targeting c11orf74, cfap20 and cep192. Targeting of cfap20 resulted in significant edema formation, and was rescued by co-injection of cfap20 mRNA. Targeting cep192 resulted in significant edema formation, but at low percentages. (e, f) Craniofacial analysis revealed that the distance between the eyes was smaller for cep192, ttc30a, and cfap20 targeted embryos, but not c11orf74. $(\mathrm{g})$ Percentage of edema formation in embryos injected at the one cell state in parallel to the animals raised to the froglet stage were used as efficacy control. (h) An example of a ttc30a mutant froglet with polydactyly (orange arrowheads) and both fore- and hindlimb malformations (red arrowheads). Embryos were injected at the 2-cell stage into the right blastomere with sgRNA targeting ttc30a and Cas9. (j) Examples of unilaterally cep192 and cfap20 targeted animals without an obvious phenotype. (k) Quantification of limb defects of unilaterally targeted froglets. Shortened limbs were only detected in ttc30a targeted animals. Error bars indicate SEM. $p>0.05$ ns (not significant); $p<0.05^{*} ; p<0.01 * * ; p<0.001 * * *$

Supplementary Figure 5: scRNA-Seq data analysis of embryonic mouse limb buds.

(a) tSNE plot of all identified cell clusters in scRNA-Seq data of E15.5 mice limbs. (b, c) Marker genes used for defining the clusters. Gene expression is indicated in blue on the tSNE-plot. (c) Violin plots of respective marker transcript levels across the assigned tissue clusters. (d) Expression of Ift80, Ift172, Ttc30a1 and Ttc30a2 depicted in blue in the tSNE plot. (e) Expression levels of Ttc30a1 and Ttc30a2 in the tissue clusters.

Supplementary Table 1

Differentially regulated transcripts detected by RNA-Seq of ift80 CRISPR targeted embryos.

Supplementary Table 2

Differentially regulated transcripts detected by RNA-Seq of ift172 CRISPR targeted embryos.

Supplementary Table 3

Resource List and references used for the in silico discovery screen.

Supplementary Table 4

Candidate genes of the in silico discovery screen for SRTD at FDR 5\%.

Supplementary Table 5

Sequences of primers and morpholino oligonucleotides.

Supplementary Table 6

Results of the in silico screen for OMIM diseases/phenotypes at FDR 5\%.

(Amyotrophic lateral sclerosis, Bardet-Biedl syndrome, cortical dysplasia, cranioectodermal dysplasia, DiamondBlackfan anemia, Joubert syndrome, Long-QT syndrome, Meckel syndrome, nephronophthisis, pachyonychia congenita, primary cilia dyskinesia, Senior-Loken syndrome) 
bioRxiv preprint doi: https://doi.orq/10.1101/2020.11.27.400994: this version posted November 27.2020 . The copyriaht holder for this

preprint (which was not certified by peer review) is the author/funder, who has granted bioRxiv a license to display the preprint in perpetuity. It is made available under aCC-BY-NC-ND 4.0 International license.

Supplementary Movie 1

Fluorescein-dextran was injected into the coelomic cavity and recorded by a timelapse movie (4fps for $3 \mathrm{~min}$ ). AVImovies are shown with 40fps. Excretion was only observed for s/c45a2 targeted embryos (embryo on top) but not for embryos targeted for SC-genes (ift80 targeted embryo in the middle, ift172 targeted embryo at the bottom).

\section{Supplementary Movie 2}

Time-lapse movie for excretion in ttc30a targeted tadpoles (s/c45a2 targeted embryo on top; ttc30a targeted embryos at the bottom). 
bioRxiv preprint doi: https://doi.org/10.1101/2020.11.27.400994; this version posted November 27, 2020. The copyright holder for this

E u prey rint (which was not certified by peer review) is the author/funder, who has granted bioRxiv a license to display the preprint in

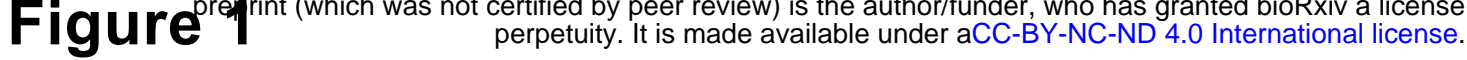

a

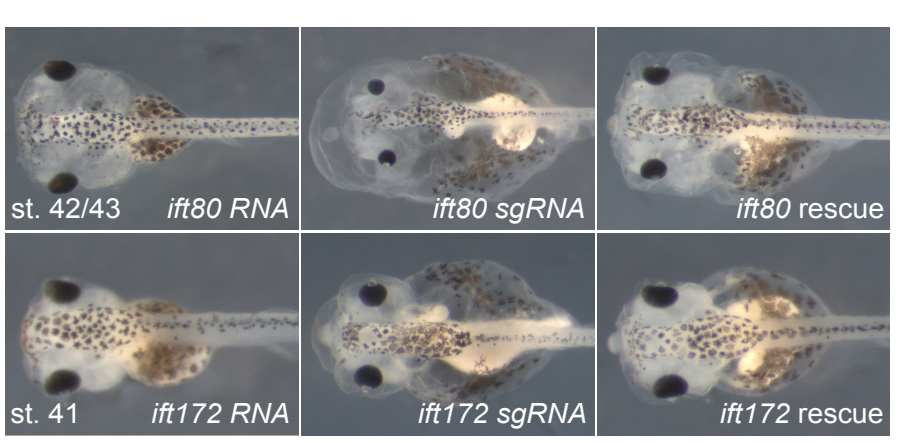

b

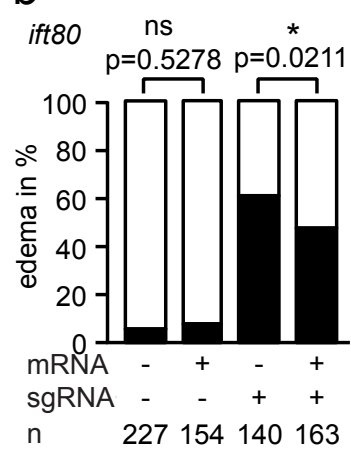

- edema $\square$ no edema

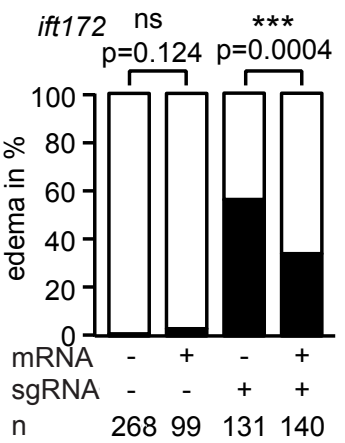

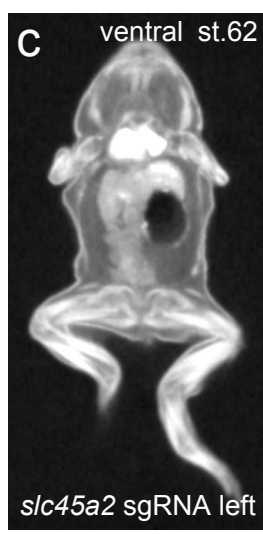
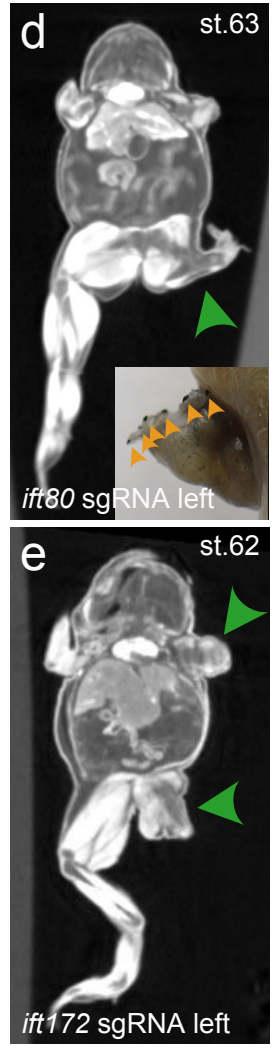
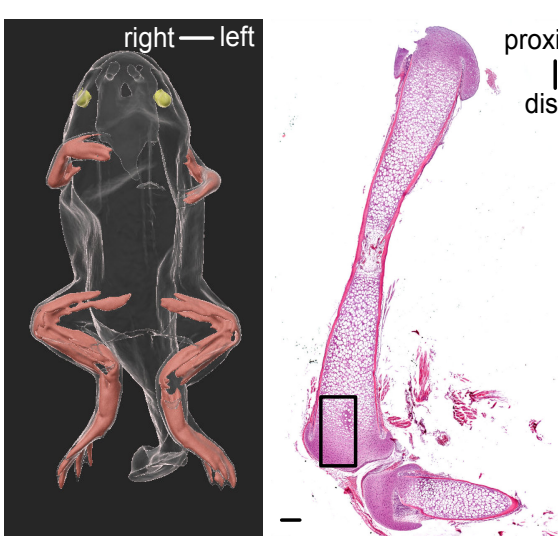

dista

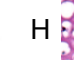

PH

$\mathrm{PH}$

P

R
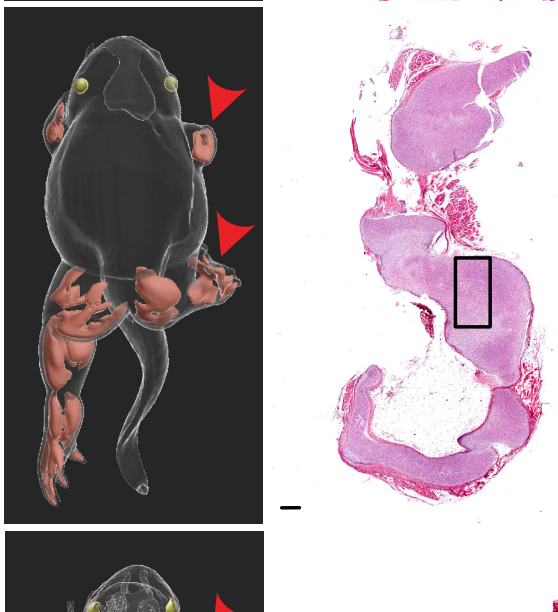
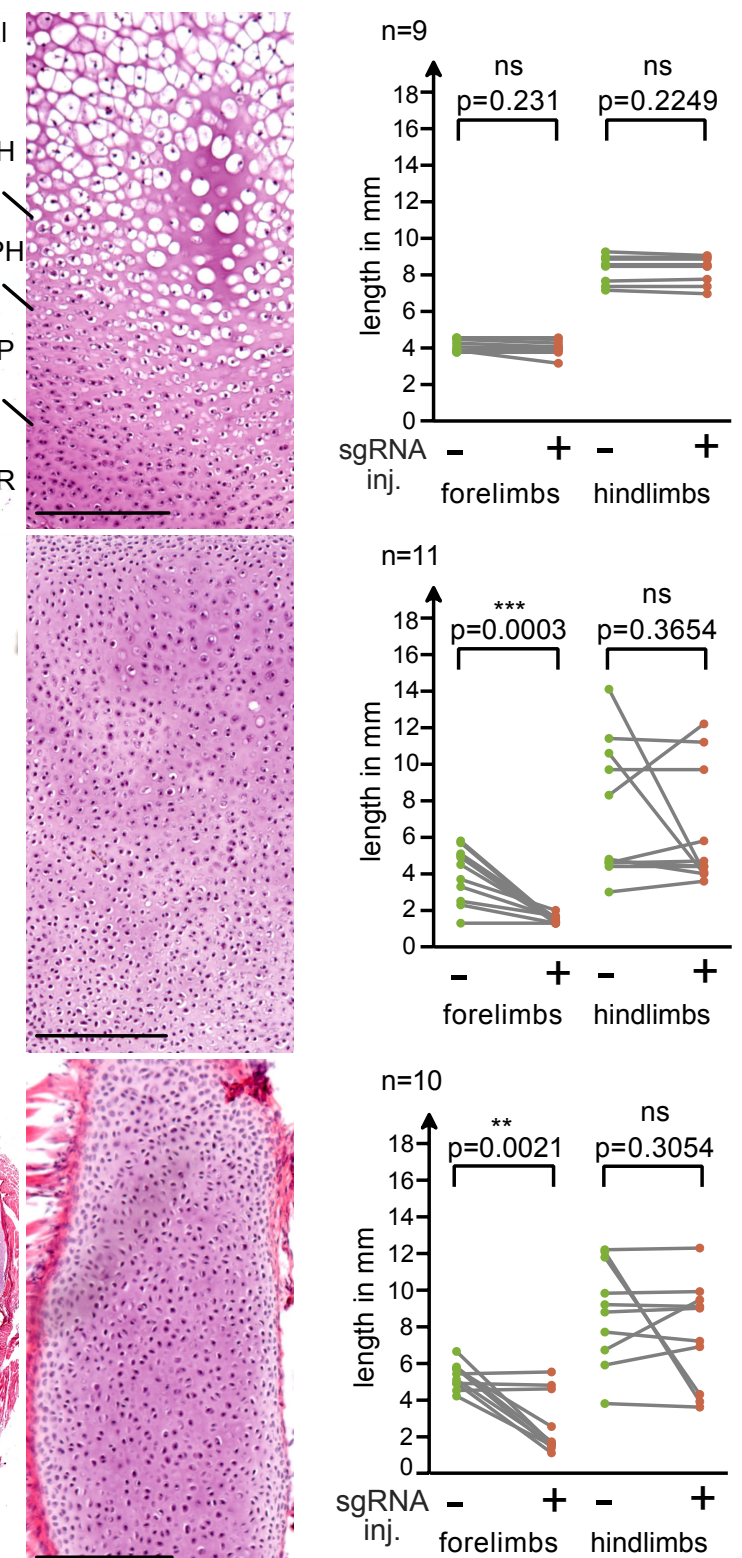

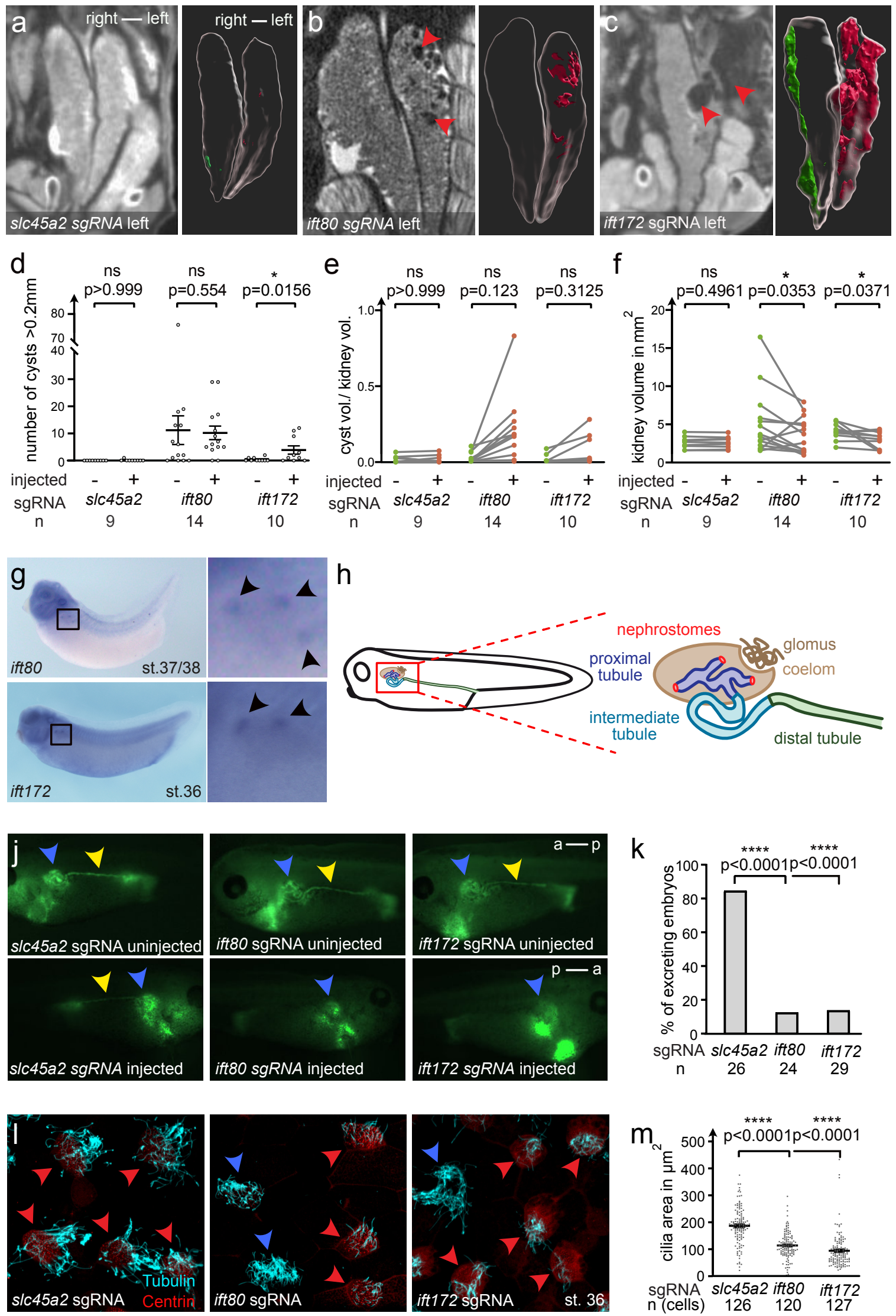
bioRxiv preprint doi: https://doi.org/10.1101/2020.11.27.400994; this version posted November 27, 2020. The copyright holder for this El 1 - 1 regint (which was not certified by peer review) is the author/funder, who has granted bioRxiv a license to display the preprint in perpetuity. It is made available under aCC-BY-NC-ND 4.0 International license.

a

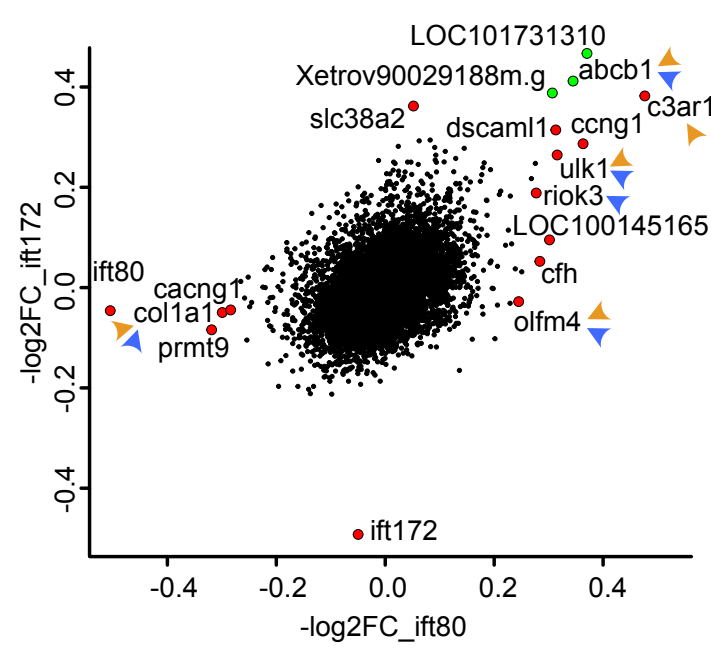

\begin{tabular}{|c|c|c|c|}
\hline Gene & Interaction with $\mathrm{Hh}$ & Interaction with Wnt & References \\
\hline$a b c b 1$ & \begin{tabular}{|l|l|} 
Gli1 activates $a b c b$ \\
expression
\end{tabular} & $\begin{array}{l}\text { B-catenin activates } a b c b \\
\text { expression }\end{array}$ & $\begin{array}{l}\text { Sims-Mourtada et al } \\
\text { 2007; } \\
\text { Chen et al. 2014; } \\
\text { Corrêa et al. } 2012\end{array}$ \\
\hline c3ar1 & & $\begin{array}{l}\text { enhances } \\
\text { signaling via } \mathrm{C} 3 \mathrm{a}\end{array}$ & $\begin{array}{l}\text { Matsumoto et al. } \\
2017\end{array}$ \\
\hline col1a1 & $\begin{array}{l}\text { Ihh } \\
\text { col1a1 }\end{array} \begin{array}{l}\text { signaling inhibits } \\
\text { expression }\end{array}$ & $\begin{array}{|llc|}\text { Col1a1 } & \text { increases } \\
\text { expression } & \text { of } & \text { Wnt/PCP } \\
\text { factors } & & \end{array}$ & $\begin{array}{l}\text { Sun et al. 2018; } \\
\text { Zhang et al. 2018 }\end{array}$ \\
\hline olfm4 & $\begin{array}{l}\text { Olfm4 reduces Shh target } \\
\text { expression (shh, ptch, } \\
\text { gli1) by binding Shh and } \\
\text { preventing } \\
\text { binding }\end{array}$ & $\begin{array}{|lr|}\text { olfm4 transcription is } \\
\text { induced by Wnt/ } \beta \text {-cat and } \\
\text { Olfm4 inhibits Wnt/ } \beta \text {-cat by } \\
\text { competitiver receptor } \\
\text { binding }\end{array}$ & $\begin{array}{l}\text { Li et al. 2015; } \\
\text { Liu et al. } 2016\end{array}$ \\
\hline riok3 & $\begin{array}{l}\text { Riok3 activates hh- } \\
\text { signaling by translocating } \\
\text { SUFU to the nucleus }\end{array}$ & $\begin{array}{l}\text { Ulk1 inhibits wnt/ß-cat by } \\
\text { Dsh phosphorylation; } \\
\text { Wnt5a induces Ulk1 kinase } \\
\text { activity }\end{array}$ & Tariki et al. 2013 \\
\hline ulk1 & $\begin{array}{l}\text { Shh promotes activation } \\
\text { (phosphorylation) of Ulk1 } \\
\text { by AMPK }\end{array}$ & & $\begin{array}{l}\text { Xiao et al. 2015; } \\
\text { Hwang et al. 2019; } \\
\text { Jati et al. 2018 }\end{array}$ \\
\hline
\end{tabular}

b $\begin{array}{cccc}\text { Input List } & \text { Resource Lists }(n=90) & \text { Enriched Lists }(n=16) & \text { Candidate List } \\ 17 \text { SC genes } & \text { proteomic, protein } & \text { SC genes sign. } & 65 \text { genes at 5\% FDR } \\ \text { interaction, functional, or } & \text { overrepresented } & \text { ranked by p-value }\end{array}$ expression screens, ...

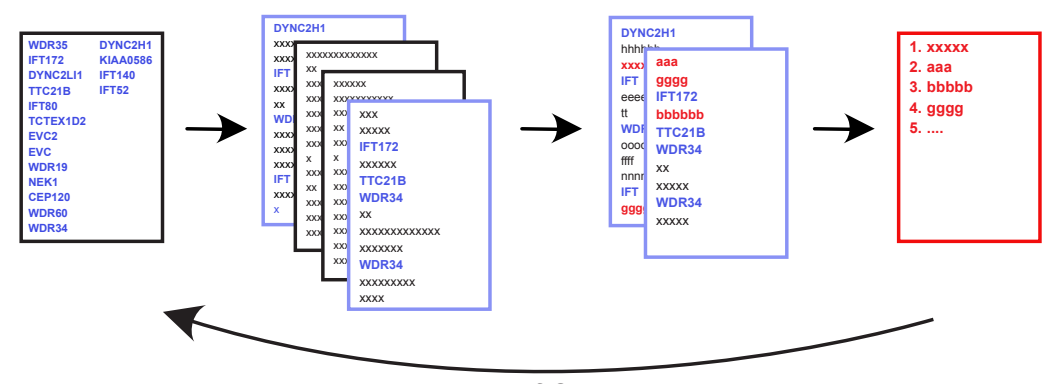

Validation of Candidates

10 million randomly drawn input lists, empirical $p$-value for each candidate: $P V a l=P\left(\sigma^{i}\right.$ final $($ input $\left.) \geq \sigma_{\text {final }(\text { resource })}\right)$

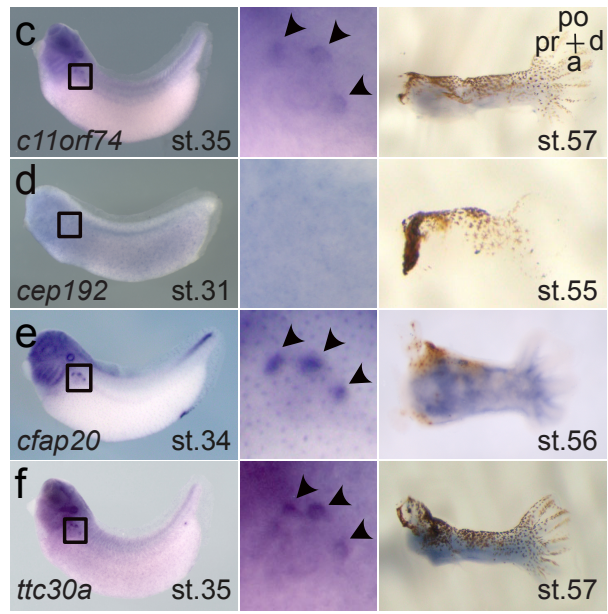

g

\begin{tabular}{|c|c|c|c|c|c|c|}
\hline c11orf74 & cep192 & cfap20 & ttc30a & Description & Species & Reference \\
\hline & & $\mathrm{x}$ & & upregulated by FoxJ1 & zebrafish & Choksi et al. 2014 \\
\hline \multirow[t]{4}{*}{$\mathrm{x}$} & & & & protein interactors of IFT140 & mouse & Toriyama et al. 2016 \\
\hline & & $\mathrm{x}$ & $\mathrm{x}$ & primary cilia proteome (IMCD3 cells) & mouse & Ishikawa et al. 2012 \\
\hline & $x$ & & $\mathrm{x}$ & siRNA screen for ciliogenesis factors (RPE1 cells) & human & Roosing et al. 2015 \\
\hline & & & $x$ & ciliary vesicle proteome & mouse & Phua et al. 2017 \\
\hline \multirow[t]{6}{*}{$\mathrm{x}$} & & & & protein interactors of TTC21B & mouse & Toriyama et al. 2016 \\
\hline & $x$ & $x$ & & IFT27 depended ciliary localization (cilia-APEX, IMCD3 cells) & mouse & Mick et al. 2015 \\
\hline & $\mathrm{x}$ & & $\mathrm{x}$ & loss-of-function intolerant (EXAC, pREC >0.9) & human & Lek et al. 2016 \\
\hline & & $\mathrm{x}$ & & up regulated during ciliogenesis & C. reinhardtii & Albee et al. 2015 \\
\hline & $x$ & & & centrosomal proteome (lymphoblastic KE37 cells) & human & Jakobsen et al. 2011 \\
\hline & $\mathrm{x}$ & & & protein interactors of INTU & mouse & Toriyama et al. 2016 \\
\hline $\mathrm{x}$ & & & & protein interactors of TULP3 & mouse & Toriyama et al. 2016 \\
\hline \multirow[t]{2}{*}{$\mathrm{x}$} & $x$ & $x$ & $x$ & enriched in ciliated cells (sc-RNA-Seq, lung) & mouse & Treutlein et al. 2015 \\
\hline & & & & protein interactors of TULP2 & mouse & Toriyama et al. 2016 \\
\hline$x$ & & $x$ & $x$ & enriched in late multicilitated cells & Xenopus & Quigley et al. 2017 \\
\hline
\end{tabular}


bioRxiv preprint doi: https://doi.org/10.1101/2020.11.27.400994; this version posted November 27, 2020. The copyright holder for this

Froprint (which was not certified by peer review) is the author/funder, who has granted bioRxiv a license to display the preprint in

Fig ure 4 prestint (which was not certified by peer review) is the author/funder, who has granted bioRxiv a license

a

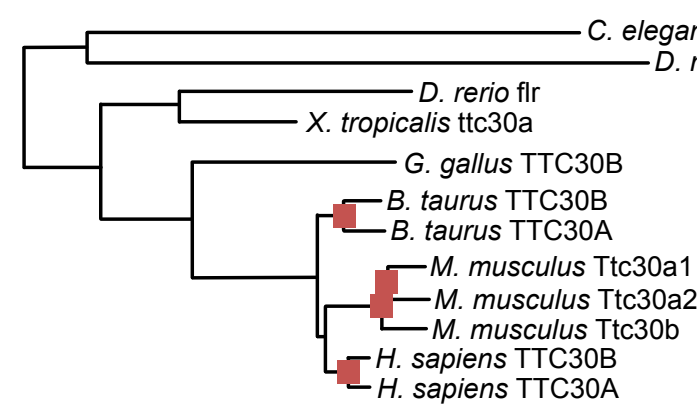

gene duplication event
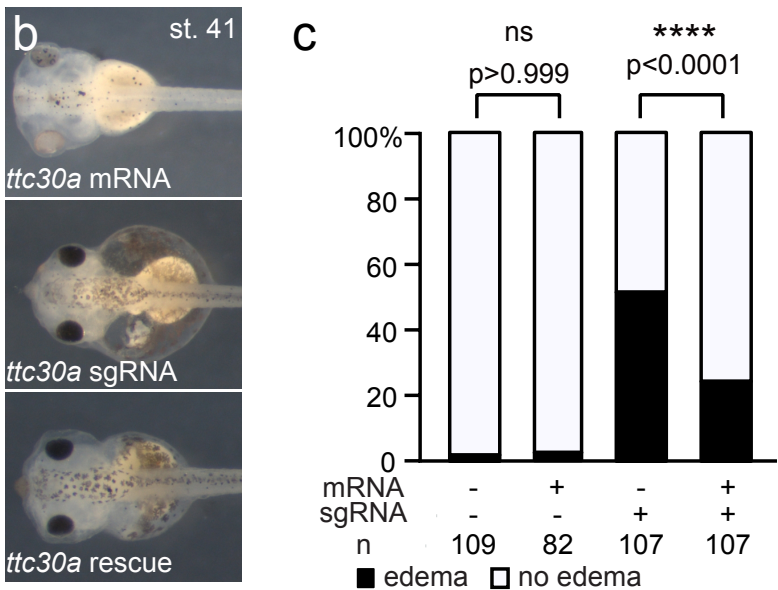
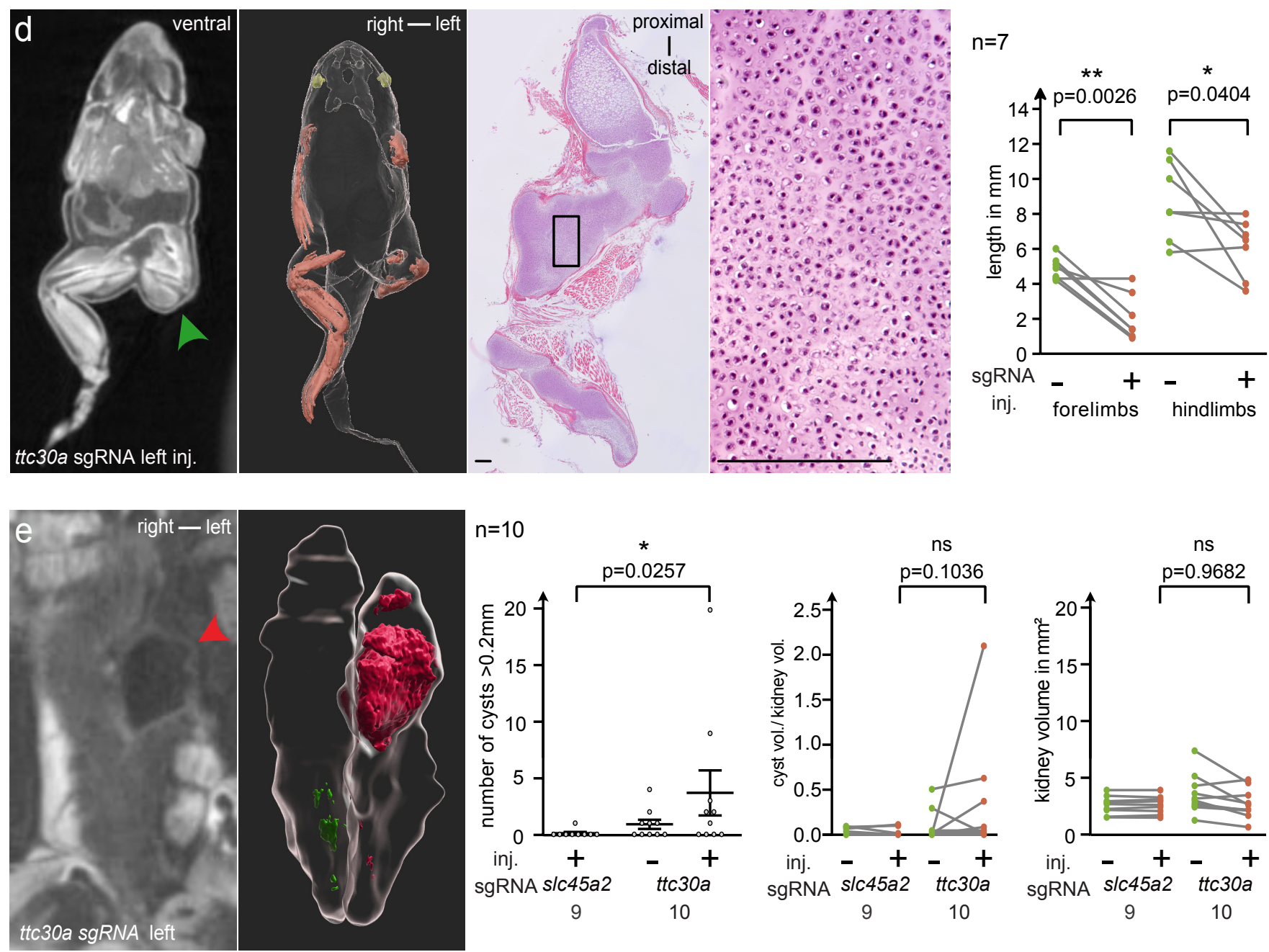

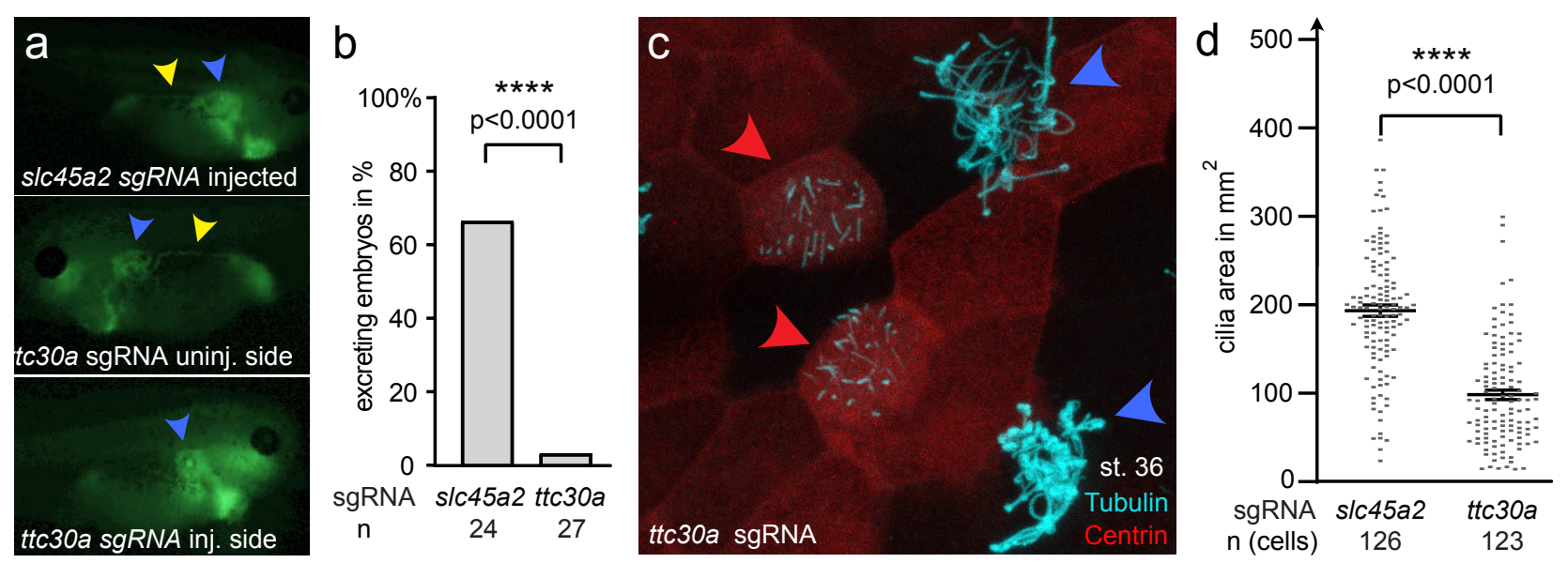

e

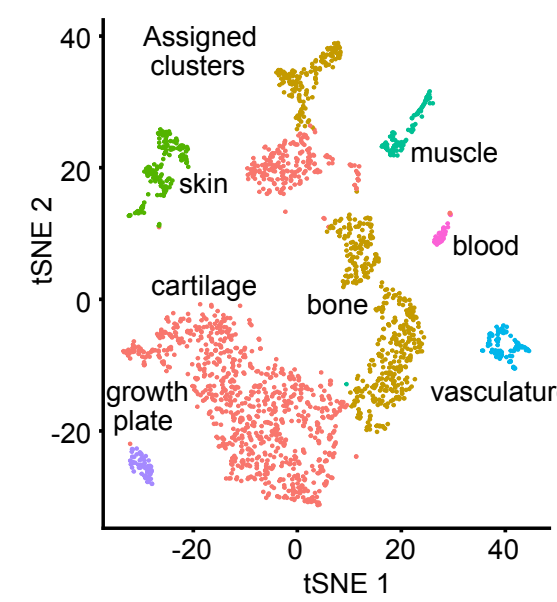

h

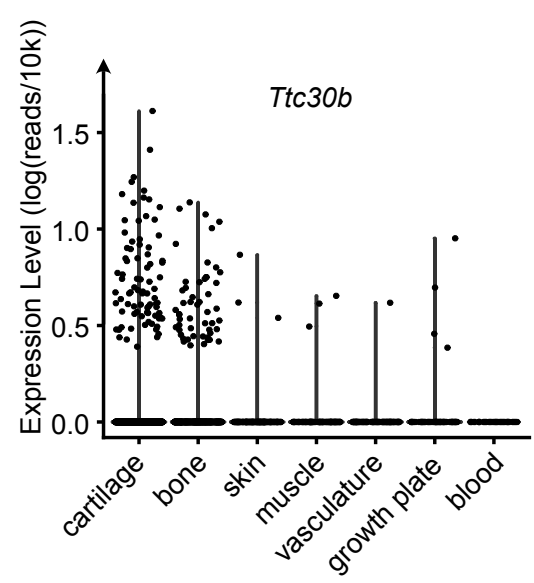

f

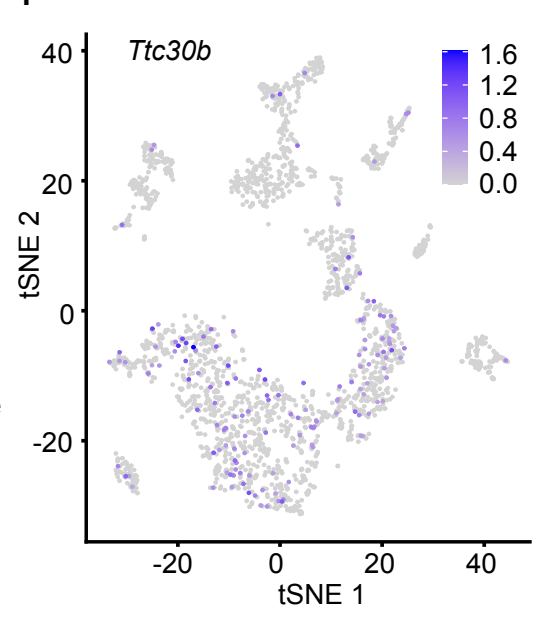

9
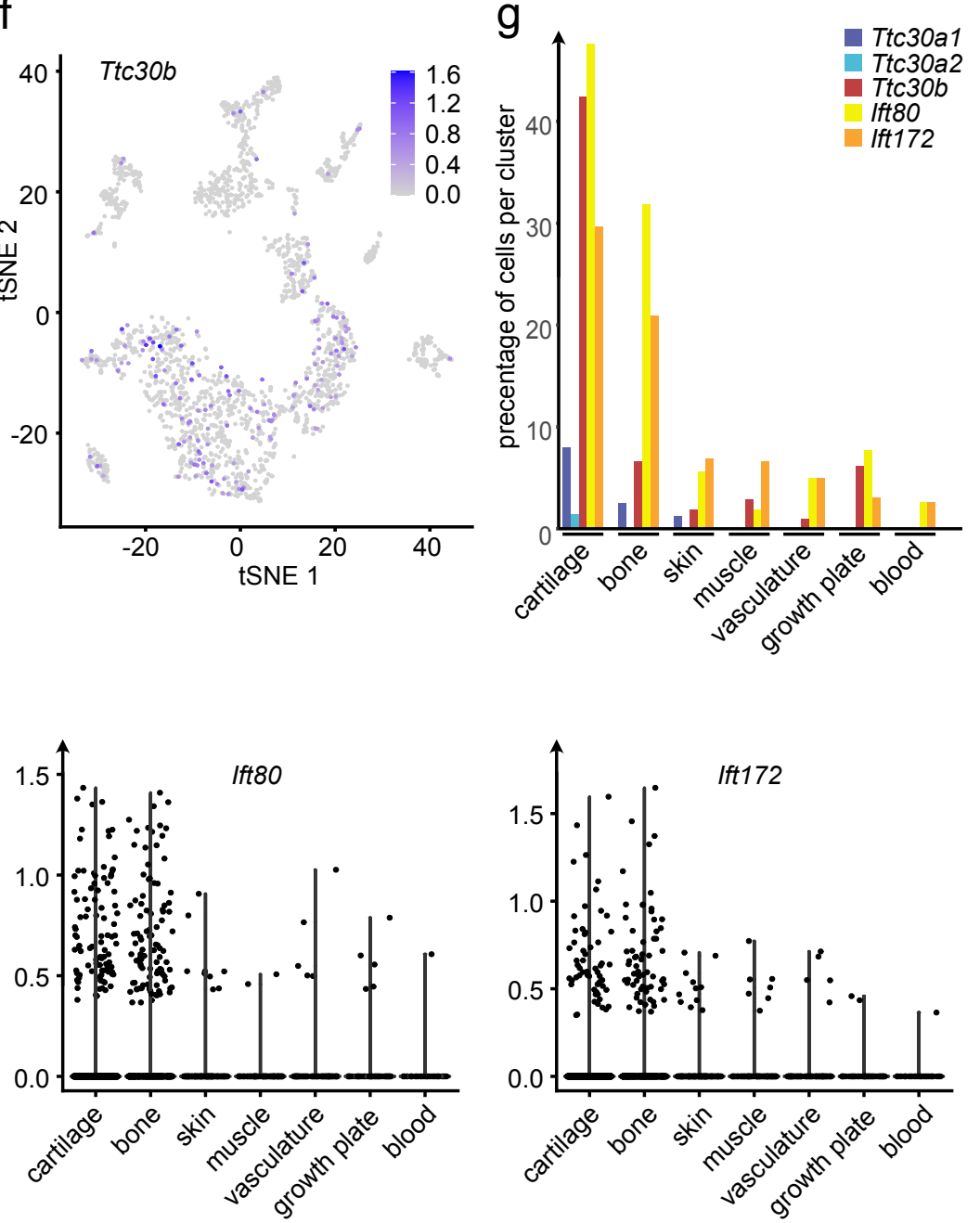